\title{
Mechanism for leptin's acute insulin-independent effect to reverse diabetic ketoacidosis
}

\author{
Rachel J. Perry, ${ }^{1}$ Liang Peng, ${ }^{1}$ Abudukadier Abulizi, ${ }^{1}$ Lynn Kennedy, ${ }^{1}$ Gary W. Cline, ${ }^{1}$ and Gerald I. Shulman ${ }^{1,2,3}$ \\ 'Department of Internal Medicine, ${ }^{2}$ Department of Cellular and Molecular Physiology, and ${ }^{3}$ Howard Hughes Medical Institute, Yale University School of Medicine, New Haven, Connecticut, USA.
}

\begin{abstract}
The mechanism by which leptin reverses diabetic ketoacidosis (DKA) is unknown. We examined the acute insulinindependent effects of leptin replacement therapy in a streptozotocin-induced rat model of DKA. Leptin infusion reduced rates of lipolysis, hepatic glucose production (HCP), and hepatic ketogenesis by 50\% within 6 hours and were independent of any changes in plasma glucagon concentrations; these effects were abrogated by coinfusion of corticosterone. Treating leptin- and corticosterone-infused rats with an adipose triglyceride lipase inhibitor blocked corticosterone-induced increases in plasma glucose concentrations and rates of HGP and ketogenesis. Similarly, adrenalectomized type 1 diabetic (T1D) rats exhibited decreased rates of lipolysis, HCP, and ketogenesis; these effects were reversed by corticosterone infusion. Leptin-induced decreases in lipolysis, HGP, and ketogenesis in DKA were also nullified by relatively small increases (15 to $70 \mathrm{pM}$ ) in plasma insulin concentrations. In contrast, the chronic glucose-lowering effect of leptin in a STZ-induced mouse model of poorly controlled T1D was associated with decreased food intake, reduced plasma glucagon and corticosterone concentrations, and decreased ectopic lipid (triacylglycerol/diacylglycerol) content in liver and muscle. Collectively, these studies demonstrate marked differences in the acute insulin-independent effects by which leptin reverses fasting hyperglycemia and ketoacidosis in a rodent model of DKA versus the chronic pleotropic effects by which leptin reverses hyperglycemia in a non-DKA rodent model of T1D.
\end{abstract}

\section{Introduction}

Recent studies have demonstrated that leptin, administered either systemically or into the cerebroventricular or hypothalamic space, can lower plasma glucose concentrations and prolong life in insulinopenic rodent models of poorly controlled type 1 diabetes (T1D) (1-4). However, the mechanism by which this occurs has been the subject of recent debate and has been attributed to suppression of hyperglucagonemia or glucagon responsiveness (1, $2,5-8)$, improvements in insulin sensitivity $(7,9)$, and activation of Creb-regulated transcription coactivator 1 signaling (10). Furthermore, even less is known regarding the mechanism by which leptin reverses ketaoacidosis in diabetic rodents. In this regard, hepatic ketogenesis is thought to be regulated mostly by malonyl $\mathrm{CoA}$, a key regulator of carnitine palmitoyl transferase-I (CPT-I) activity, which in turn regulates fatty acid transport into the mitochondria (11). However, until recently, the small pool size and lability of hepatic malonyl CoA have prevented direct measurements of malonyl CoA concentrations, making it difficult to examine the role of malonyl CoA in ketogenesis as well as to determine whether leptin-induced increases in hepatic malonyl CoA concentrations are responsible for leptin's ability to reverse ketoacidosis in rodent models of diabetic ketoacidosis (DKA). In a recent study, we found that acute leptin replacement therapy reduces

\section{Related Commentary: p. 450}

Conflict of interest: The authors have declared that no conflict of interest exists. Submitted: May 9, 2016; Accepted: October 27, 2016

Reference information: J Clin Invest. 2017;127(2):657-669.

https://doi.org/10.1172/JCl88477. plasma glucose concentrations by suppressing plasma corticosterone concentrations, resulting in lower rates of lipolysis, which in turn leads to reductions in hepatic acetyl CoA content and hepatic gluconeogenesis in 3 rat models of poorly controlled diabetes (12). However, the role of leptin-mediated suppression of hypercorticosteronemia as necessary or sufficient to mediate leptin's acute, insulin-independent effect in T1D has recently been called into question (13) by Morton et al. They found that leptin as well as glucocorticoid receptor blockade had no effect on plasma glucose concentrations in a streptozotocin-treated (STZ-treated) rat model of T1D and that adrenalectomized T1D rats did not exhibit lower plasma glucose concentrations than nonadrenalectomized T1D rats. From these results, they concluded that lowering plasma corticosterone concentrations is neither necessary nor sufficient to mediate leptin's effects to lower plasma glucose concentrations in T1D rodents. However, there are major differences in the STZ-treated T1D rat model that Morton et al. used in their studies compared with the STZ-treated T1D rat model of DKA used in our studies, which could account for these different results. Specifically, Morton et al. used rats that are less insulinopenic than ours and performed mostly chronic studies in fed rats, whereas we performed primarily acute experiments in fasted rats with 5 -fold lower plasma insulin concentrations.

In order to further characterize the mechanism by which leptin replacement therapy acutely reverses both the increased rates of hepatic glucose production and hepatic ketogenesis associated with DKA and to understand the reasons for the disparate findings between our study and that of Morton et al., we performed a series of experiments to assess the mechanisms for the effects of leptin replacement therapy on hyperglycemia and ketoacidosis under sev- 
eral conditions. First, in order to examine the mechanism by which leptin replacement therapy acutely reverses DKA in an insulinindependent manner, we examined the effects of leptin treatment on rates of lipolysis, hepatic glucose production, ketogenesis, hepatic acetyl CoA, and malonyl CoA content in a rat model of STZinduced DKA. Secondly, to determine whether lipolysis mediates increases in hepatic glucose production (HGP) and ketogenesis, we treated leptin- and corticosterone-infused rats in DKA with a small molecule inhibitor of adipose triglyceride lipase (ATGL). Next, in order to examine the role of corticosterone in driving DKA, we assessed rates of lipolysis, ketogenesis, and HGP in an adrenalectomized STZ-induced diabetic rat model with and without corticosterone replacement in both the fed and fasted state. So as to further characterize the impact of insulin and feeding on these leptinmediated effects, we then examined the acute effect of leptin replacement therapy on glucose turnover and ketogenesis in DKA rats when plasma insulin concentrations were increased from approximately $15 \mathrm{pM}$ to approximately $70 \mathrm{pM}$ by an infusion of insulin under both fed and fasted conditions. Finally, in order to examine the mechanism or mechanisms by which chronic leptin replacement therapy reduces blood glucose concentrations, we evaluated the effects of chronic leptin replacement therapy on plasma glucose, glucagon, and glucocorticoid concentrations as well as food intake, energy expenditure, and ectopic lipid content in liver and skeletal muscle in a nonketoacidotic STZ-induced mouse model of T1D.

\section{Results}

Leptin acutely suppresses lipolysis and HGP in an insulin-independent manner by suppression of the hypothalamic-pituitary-adrenal axis. STZ-treated rats were hyperglycemic $(\sim 400 \mathrm{mg} / \mathrm{dl})$ and severely insulinopenic $(\sim 15 \mathrm{pM})$ after an overnight fast (Figure 1, A and B) as compared with nondiabetic controls, which exhibited plasma glucose concentrations of $106 \pm 2 \mathrm{mg} / \mathrm{dl}$ and plasma insulin concentrations of $56 \pm 5 \mathrm{pM}$. Consistent with our previous study (12) and that of Morton et al. (13), poorly controlled STZ-induced diabetic rats exhibited low plasma leptin concentrations (0.16 \pm $0.01 \mathrm{vs} .1 .02 \pm 0.16 \mathrm{ng} / \mathrm{ml}$ in nondiabetic controls) and increased plasma corticosterone concentrations $(245 \pm 43 \mathrm{vs} .94 \pm 3 \mathrm{ng} / \mathrm{ml}$ in nondiabetic controls) compared with normal rats not treated with STZ (Figure 1, C and D). Administering a 6-hour intraarterial infusion of leptin at a dose to match plasma leptin concentrations in normal rats suppressed plasma corticosterone concentrations by approximately 60\% (Figure 1, C and D). This effect was centrally mediated, as demonstrated by an approximately $50 \%$ reduction in plasma adrenocorticotropic hormone (ACTH) concentrations (Figure 1E), approaching those of nondiabetic controls $(4.8 \pm 0.6$ $\mathrm{pg} / \mathrm{ml})$. In contrast, there were no differences in plasma glucagon, epinephrine, norepinephrine, growth hormone, or IGF-1 concentrations (Figure 1, F and G, and Supplemental Figure 1, A-C; supplemental material available online with this article; https:// doi.org/10.1172/JCI88477DS1). In order to understand the mechanism by which leptin acutely lowers plasma glucose in T1D rats, we measured rates of HGP and found that a 6-hour leptin infusion lowered HGP rates by more than $40 \%$ (Figure $1 \mathrm{H}$ ). However, leptin's acute ability to reverse hyperglycemia and to lower HGP was prevented by coinfusing leptin-treated T1D rats with corticosterone to restore their plasma corticosterone concentrations to those measured in control T1D rats. This intervention increased plasma glucose concentrations and HGP to levels similar to those measured in control T1D rats without affecting plasma insulin, glucagon, epinephrine, norepinephrine, growth hormone, or IGF-1 concentrations (Figure 1, A-H, and Supplemental Figure 1, A-C).

Next, to determine the cause of the increased endogenous glucose production measured in T1D rats, we measured plasma nonesterified fatty acid (NEFA) and $\beta$-hydroxybutyric acid ( $\beta \mathrm{OHB}$ ) concentrations and found both to be elevated in T1D rats as compared with nondiabetic controls $(0.94 \pm 0.11 \mathrm{mEq} / 1 \mathrm{NEFA}$ and $0.76 \pm 0.10$ $\mathrm{mM} \beta \mathrm{OHB}$ in normal rats not treated with STZ), with leptin suppressing both NEFA and $\beta \mathrm{OHB}$ concentrations, and corticosterone infusion acutely restoring each to that measured in T1D rats (Figure $2, \mathrm{~A}$ and $\mathrm{B})$. We next confirmed that our T1D control rats were in DKA, measuring an anion gap $40 \%$ greater in T1D rats than in nondiabetic rats $(17 \pm 2 \mathrm{mEq} / \mathrm{l})$, and that DKA was reversed with leptin treatment, but not with leptin and corticosterone infusion (Figure 2C). To assess the potential role of increased lipolysis in mediating the glucose- and ketone-lowering effects of leptin, we performed a steady-state infusion of $\left[{ }^{13} \mathrm{C}_{16}\right]$ palmitate and $\left[1,1,2,3,3-{ }^{2} \mathrm{H}_{5}\right]$ glycerol and, consistent with our previous study (12), we found that leptin lowered rates of lipolysis in T1D rats, but replacement corticosterone abrogated this effect (Figure 2, D and E). Leptin-treated rats also exhibited suppression of ketogenesis, as demonstrated by a $50 \%$ reduction in the rate of $\beta \mathrm{OHB}$ turnover (Figure $2 \mathrm{~F}$ ). T1D rats exhibited decreased hepatic acetyl CoA content, but there were no changes in hepatic malonyl CoA compared to non diabetic control rats (Non diabetic control rats; hepatic acetyl-CoA: $96 \pm 5 \mathrm{nmol} / \mathrm{g}$, malonyl-CoA: $0.51 \pm 0.08 \mathrm{nmol} / \mathrm{g}$ ), and short-term leptin treatment was associated with suppression of hepatic acetyl CoA content, but no changes in hepatic malonyl CoA content (Figure 2, G and $\mathrm{H}$ ).

Increased rates of lipolysis promote increased rates of HGP and ketogenesis in DKA. In order to determine the physiological role of increased lipolysis in driving hyperglycemia and ketoacidosis in DKA, we next treated a group of leptin- and corticosterone-infused DKA rats with atglistatin, a small-molecule ATGL inhibitor $(14,15)$, prior to infusion with leptin and corticosterone. This intervention lowered rates of endogenous glucose production, lipolysis, and ketogenesis as well as hepatic acetyl CoA content similar to those measured in leptin-treated DKA rats, without affecting plasma glucagon, epinephrine, norepinephrine, growth hormone, IGF-1, hepatic malonyl CoA concentrations (Figure 1, A-H, Figure 2, A-H, and Supplemental Figure $1, \mathrm{~A}-\mathrm{C}$ ). In order to better understand the roles of hepatic acetyl CoA and malonyl CoA in mediating leptin's acute effects to reverse DKA, we coinfused acetate in leptin-treated DKA rats to raise hepatic acetyl CoA concentrations to those measured in untreated rats in DKA (Figure 3, A-D). Acetate infusion increased plasma glucose concentrations and HGP 2-fold (Figure 3, $\mathrm{E}$ and F) (15). Interestingly, the 3-fold increase in hepatic acetyl CoA concentrations induced by the acetate infusion was also associated with a striking increase in ketosis, demonstrated by a doubling in both plasma $\beta \mathrm{OHB}$ concentrations and whole-body $\beta \mathrm{OHB}$ turnover (Figure 3, G and $\mathrm{H}$ ), without any change in hepatic malonyl CoA content (leptin-treated rats $0.54 \pm 0.15$, leptin plus acetate-treated rats $0.35 \pm 0.08$, leptin $\rightarrow$ leptin plus acetate-treated rats $0.37 \pm 0.16$ $\mathrm{nmol} / \mathrm{g}$; arrow denotes rats that were treated with leptin alone for 4 hours, then infused with leptin plus acetate for the final 2 hours). 
A
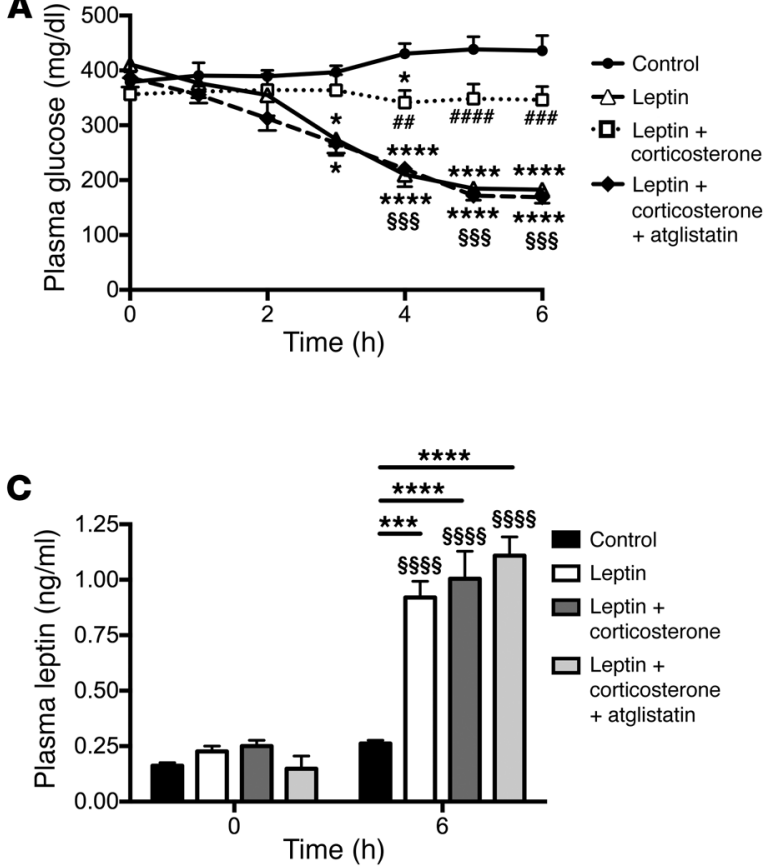

E

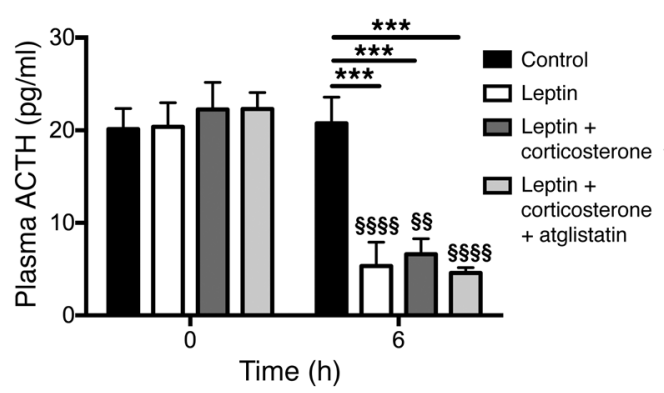

G

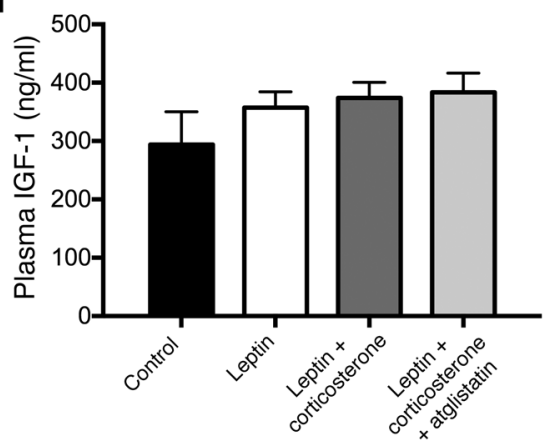

B
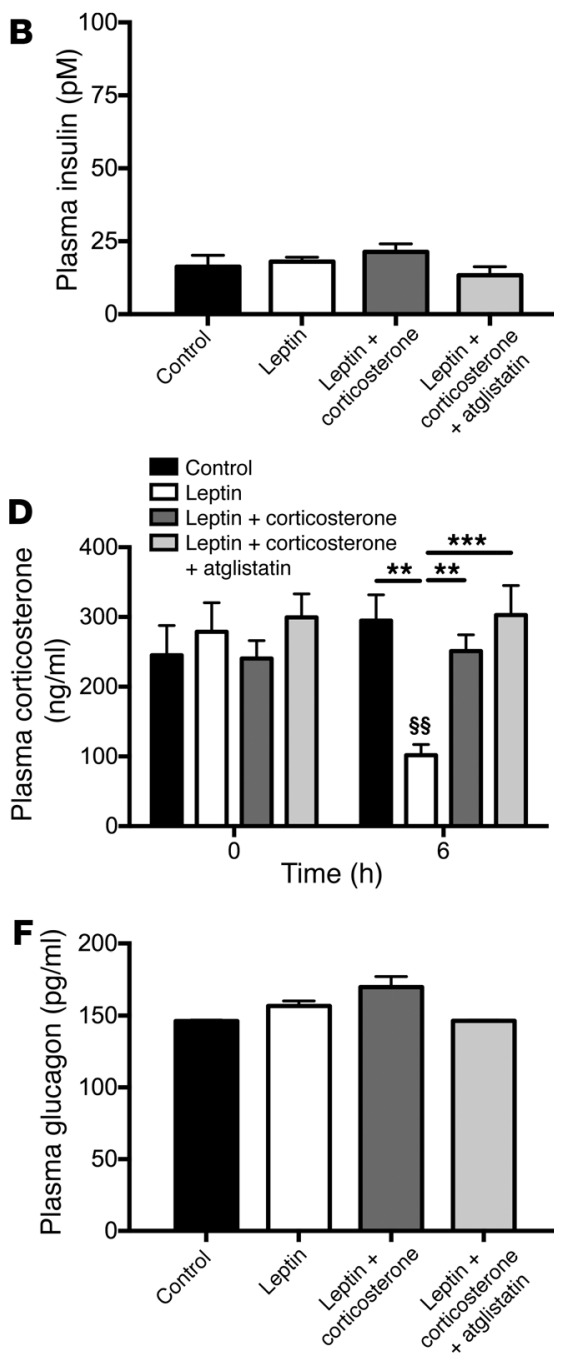

H

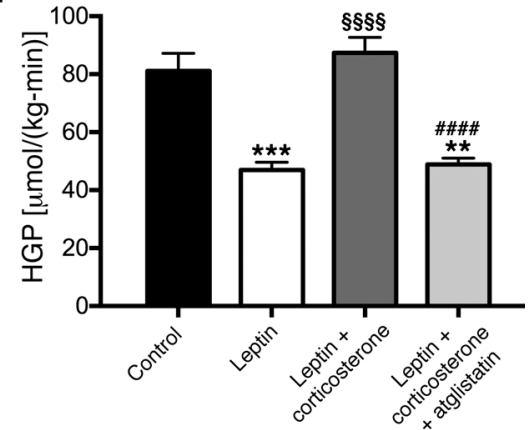

Figure 1. Leptin suppression of hypercorticosteronemia is required to mediate its glucoselowering effects by suppressing lipolysis in DKA. (A) Plasma glucose during a 6-hour acute infusion of saline (control), leptin, or leptin and corticosterone with or without pretreatment with atglistatin. ( $\mathbf{A}$ and $\mathbf{H}){ }^{*} P<0.05$ ${ }^{* *} P<0.01,{ }^{* *} P<0.001$, ${ }^{* * * *} P<0.0001$ vs. controls $\S \S \S<0.001, \S \S \S \S<0.0001$ vs. leptin-treated rats; and ${ }^{\# \#} P<0.01$, \#\#\# $P<0.001$, \#\#\#\# $P<$ 0.0001 vs. leptin plus corticosterone-treated rats. (B) Fasting plasma insulin. (C-E) Plasma leptin, corticosterone, and ACTH concentrations at 0 and 6 hours of the infusion. ${ }^{* *} P<0.01$, ${ }^{* *} P<0.001,{ }^{* * * *} P<0.0001$ between the groups indicated; $\$ \S P<0.01, \$ \S \S \S<0.0001$ vs. the same group at time zero. ( $\mathbf{F}$ and G) Fasting plasma glucagon and IGF-1 concentrations. (H) HGP after 6 hours. In all panels, data were compared by 1-way ANOVA with Bonferroni's multiple comparisons test, with data presented as the mean \pm SEM of $n=7$ (control), $n=7$ (leptin), $n=10$ (leptin plus corticosterone), and $n=6$ (leptin plus corticosterone plus atglistatin) rats.
Adrenalectomized T1D rats are protected from fasting, but not postprandial hyperglycemia. In order to further assess the role of hypercorticosteronemia in driving fasting hyperglycemia and DKA, we studied adrenalectomized STZ-treated rats in DKA. Despite low or absent corticosterone, epinephrine, and norepinephrine concentrations and glucagon, growth hormone, and IGF-1 concentrations that were not different from those of STZ-treated T1D controls, adrenalectomized rats were hyperglycemic when given water containing sucrose and corticosterone. However, both plasma NEFA and $\beta \mathrm{OHB}$ concentrations as well as the anion gap were lower in adrenalectomized rats as compared with diabetic controls, mirroring the plasma corticosterone data (Figure 4, A-I, and Supplemental Figure $2, A^{-} \mathrm{C}$ ). Futhermore, within 1 hour of removing the sucrose and corticosterone from their drinking water, adrenalectomized T1D rats exhibited plasma glucose concentrations lower than those of intact controls, and their plasma glucose concentrations and wholebody glucose, palmitate, glycerol, and $\beta \mathrm{OHB}$ turnover rates rapidly decreased and normalized within 4 hours of removing the sucrose and corticosterone drinking water (Figure 4, A and J-M). The lower rates of lipolysis and ketogenesis in adrenalectomized rats were associated with a $50 \%$ reduction in hepatic acetyl CoA content, but no change in malonyl CoA content (Figure 4, $\mathrm{N}$ and $\mathrm{O}$ ). 
A

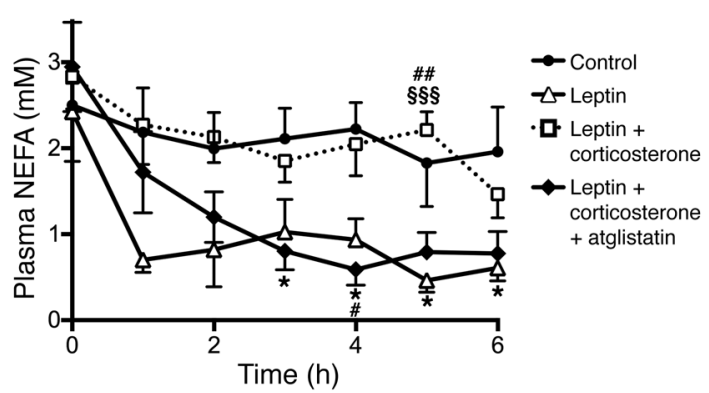

C
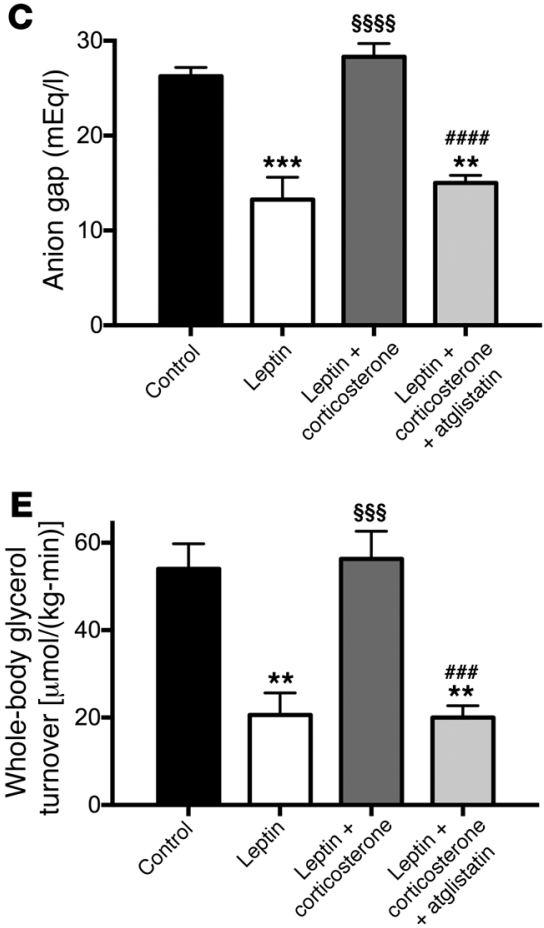

G

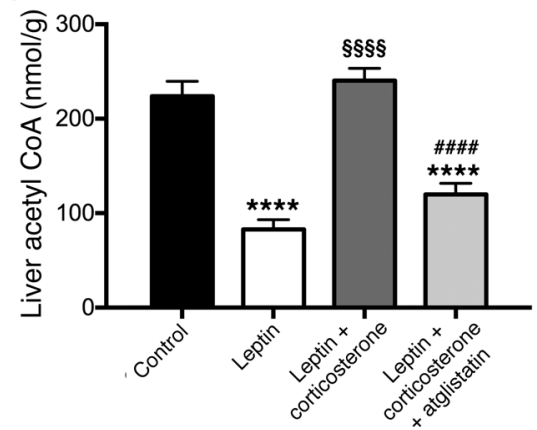

B
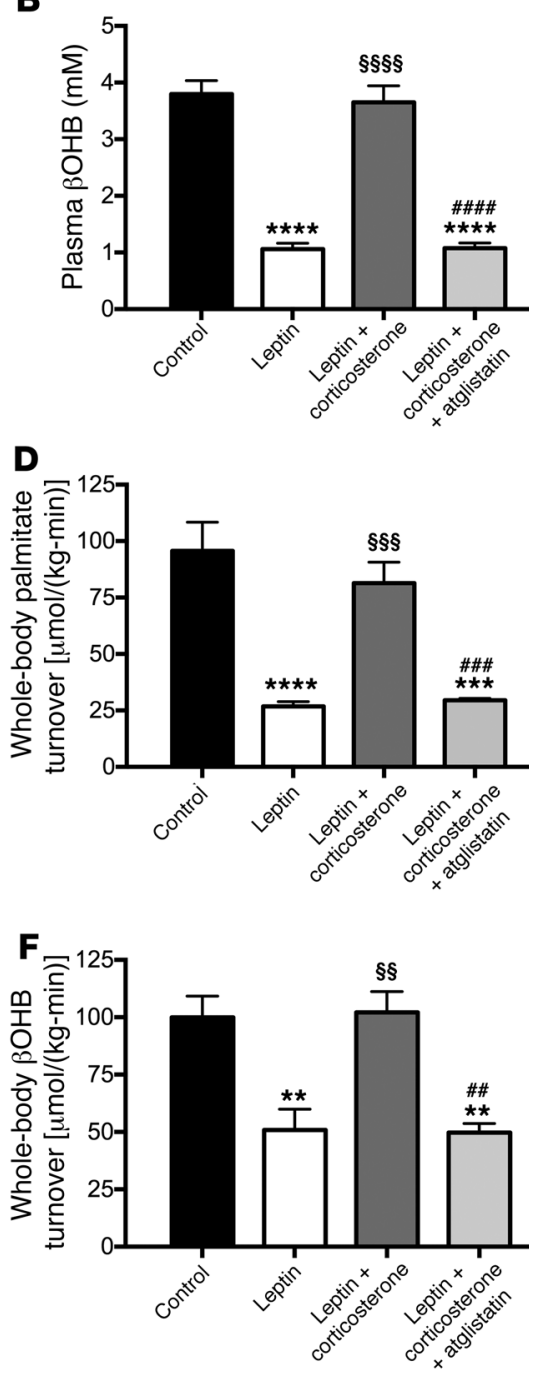

H

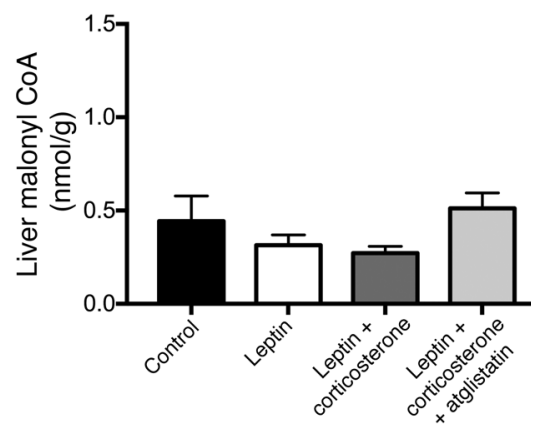

Figure 2. Suppression of lipolysis reverses hyperglycemia and ketoacidosis in T1D rats. (A) Plasma NEFA

during a 6-hour acute infusion of saline (control), leptin, or leptin and corticosterone with or without pretreatment with atglistatin. (B and C) Plasma $\beta O H B$ concentrations and anion gap after 6 hours of infusion. (D-F) Whole-body palmitate, glycer$\mathrm{ol}$, and $\beta \mathrm{OHB}$ turnover at the end of the 6-hour infusion. ( $\mathbf{G}$ and $\mathbf{H}$ ) Liver acetyl and malonyl CoA content. ${ }^{*} P<0.05,{ }^{* *} P<0.01,{ }^{* * *} P<0.001$, **** $P<0.0001$ vs. controls; ${ }^{\S} P<0.01,{ }^{\$ \$ \$} P<0.001,{ }^{\$} \$ \S \S P<0.0001$ vs. leptin-treated rats; ${ }^{\#} P<0.05$, ${ }^{\#} P<0.01,{ }^{\# \#} P<0.001$, and \#\#\# $P<0.0001$ vs. leptin plus corticosterone-treated rats by 1-way ANOVA with Bonferroni's multiple comparisons test, with data presented as the mean \pm SEM of $n=7$ (control), $n=7$ (leptin), $n=10$ (leptin plus corticosterone), and $n=6$ (leptin plus corticosterone plus atglistatin) rats.
In order to examine the role of hypercorticosteronemia in promoting hyperglycemia in DKA, we studied adrenalectomized rats infused with replacement corticosterone and found that normalizing plasma corticosterone to concentrations measured in intact T1D rats in DKA increased lipolysis, HGP, and ketoacidosis to levels similar to those measured in control T1D animals (Figure 4, A-O, and Supplemental Figure 3, A-C). Interestingly, plasma epinephrine, likely derived from extraadrenal sources (i.e., organ of Zuckerkandl), increased in the corticosterone-treated adrenalectomized animals to concentrations similar to those measured in the control animals. Finally, to confirm the role of sucrose water in promoting hyperglycemia in adrenalectomized rats, as suggested by the time course of plasma glucose, we studied adrenalectomized rats given ad libitum access to sucrose in the drinking water. Allowing the rats to continue consuming sucrose water resulted in maintenance of hyperglycemia and increased rates of whole-body glucose turnover (likely derived from both endogenous glucose production and exogenous sucrose consumption) as compared with adrenalectomized rats given water without sucrose (Figure 4, A-O). 

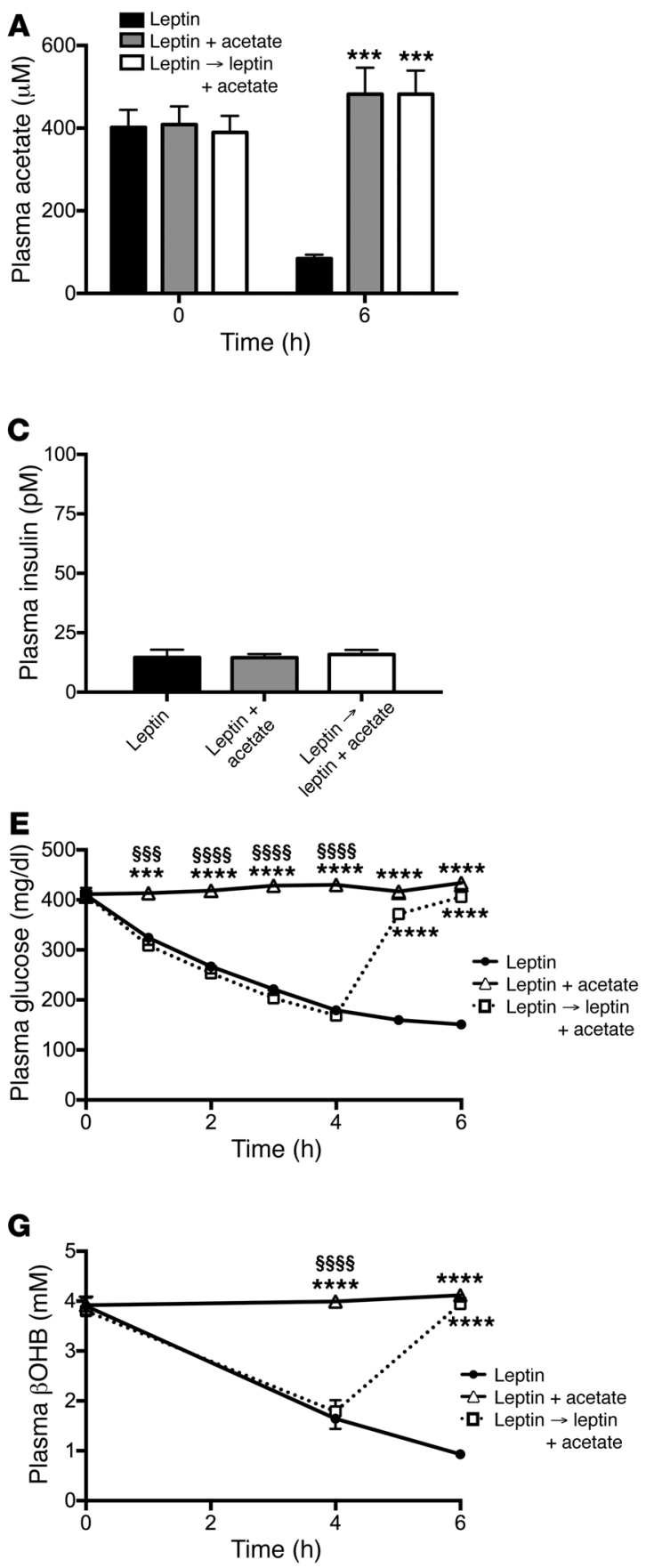
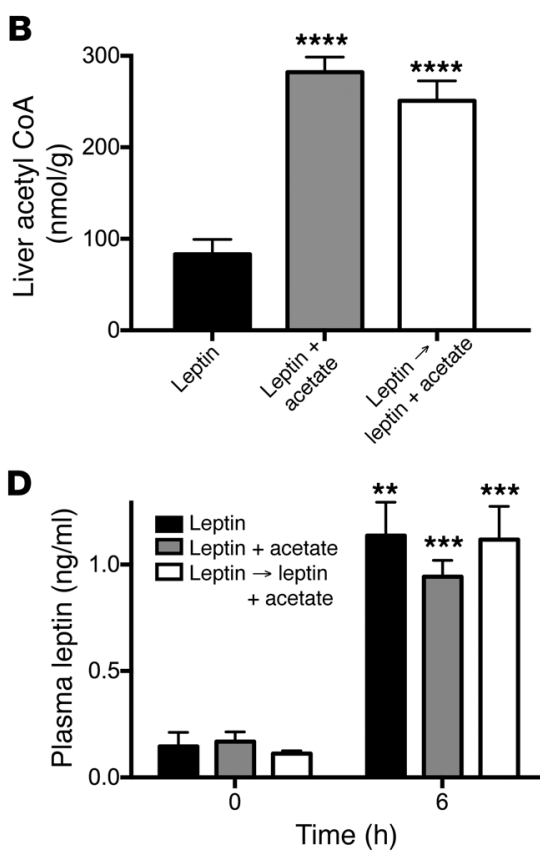

Figure 3. Suppression of hepatic acetyl CoA accounts for leptin's ability to reverse DKA. (A and B) Plasma acetate and liver acetyl CoA. (C and D) Plasma insulin and leptin. (E and $\mathbf{F})$ Plasma glucose concentrations and HGP. (G and $\mathbf{H}$ ) Plasma $\beta O H B$ concentrations and whole-body $\beta \mathrm{OHB}$ turnover. In all panels, ${ }^{*} P<0.05$, ${ }^{* *} P<0.01$, ${ }^{* *} P<0.001$, ${ }^{* * *} P<0.0001$ vs. leptin-treated rats; ${ }^{\S \S} P<0.001$, $\$ \S \S 8<0.0001$ vs. leptin plus acetatetreated rats. Data are the mean \pm SEM of $n=6$ (leptin), $n=7$ (leptin plus acetate), and $n=7$ (leptin $\rightarrow$ leptin plus acetate) per group, with data compared by ANOVA with Bonferroni's multiple comparisons test. Arrow denotes rats that were treated with leptin alone for 4 hours, then infused with leptin plus acetate for the final 2 hours.
F

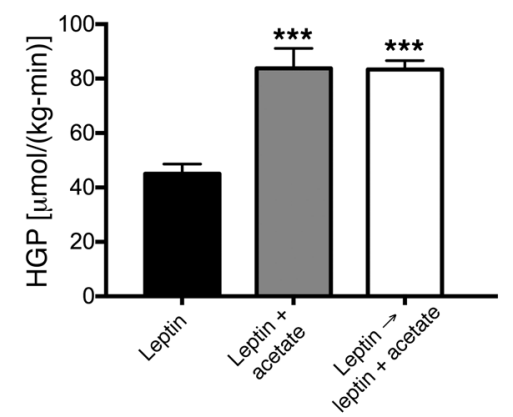

H

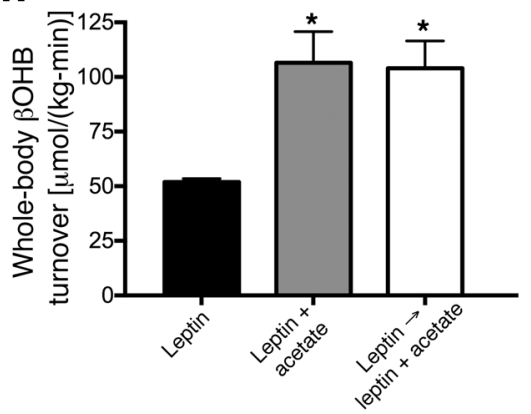

Increasing plasma insulin concentrations from 15 to $70 \mathrm{pM}$ nullifies the effect of leptin to reduce plasma glucose and ketone concentrations. In order to examine whether differences in plasma insulin concentrations in the STZ-T1D rat models could account for differences between our study and that of Morton et al., we next compared STZ-treated rats with plasma insulin concentrations of approximately $15 \mathrm{pM}$, as measured in our T1D rats in DKA, to those with plasma insulin of approximately $70 \mathrm{pM}$, as measured in Morton et al.'s study (13). We found that, while both are significantly hyperglycemic, the latter model has significant $\beta$ cell reserve, demonstrated by 3- to 6-fold higher plasma insulin and 100 to 150 $\mathrm{mg} / \mathrm{dl}$ lower plasma glucose concentrations throughout a glucose tolerance test (Figure 5, A and B). The milder insulinopenia exhibited by the diabetic rats with fasting plasma insulin concentrations of approximately $70 \mathrm{pM}$ was also associated with lower plasma $\beta O H B$ concentrations and a normal anion gap (Supplemental Figure 3 , A and B). To verify whether these associations demonstrated a causative effect of insulin to suppress ketoacidosis, even at insufficient concentrations to prevent fasting hyperglycemia, we infused rats in DKA with insulin to raise their plasma insulin concentrations from approximately $15 \mathrm{pM}$ to approximately $70 \mathrm{pM}$. Our data demonstrate that increasing plasma insulin concentrations from approximately $15 \mathrm{pM}$ to approximately $70 \mathrm{pM}$ lowered plasma glucose, NEFA, $\beta \mathrm{OHB}$, and anion gap, with leptin infusion 
A

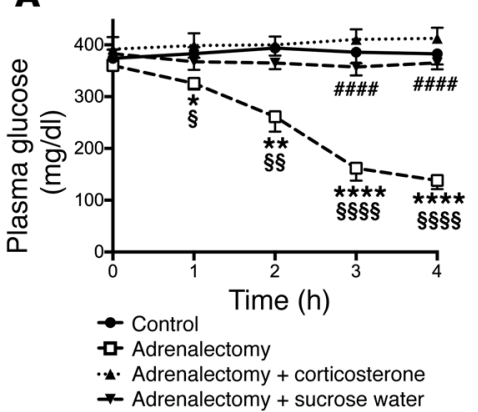

D

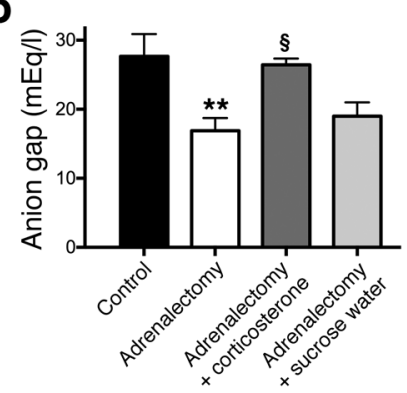

G
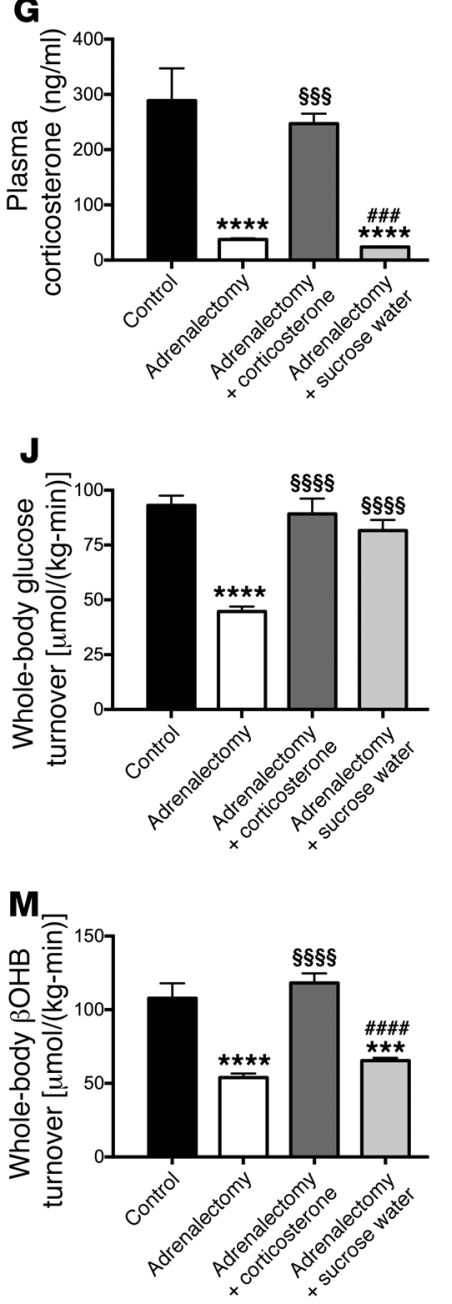

B
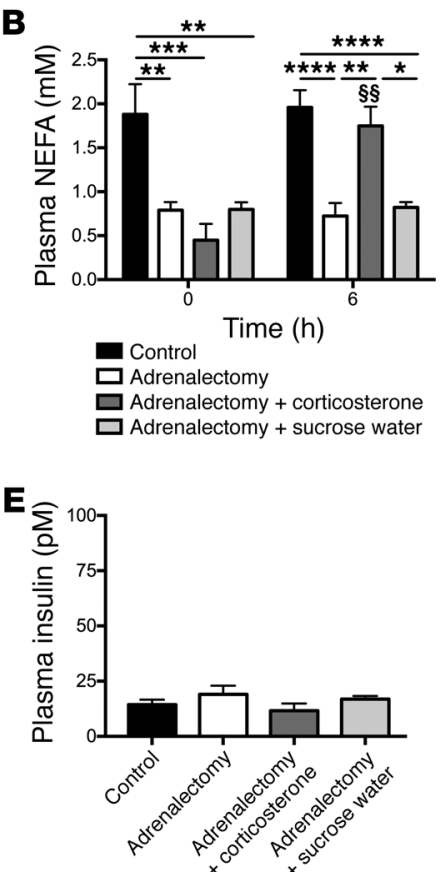

H
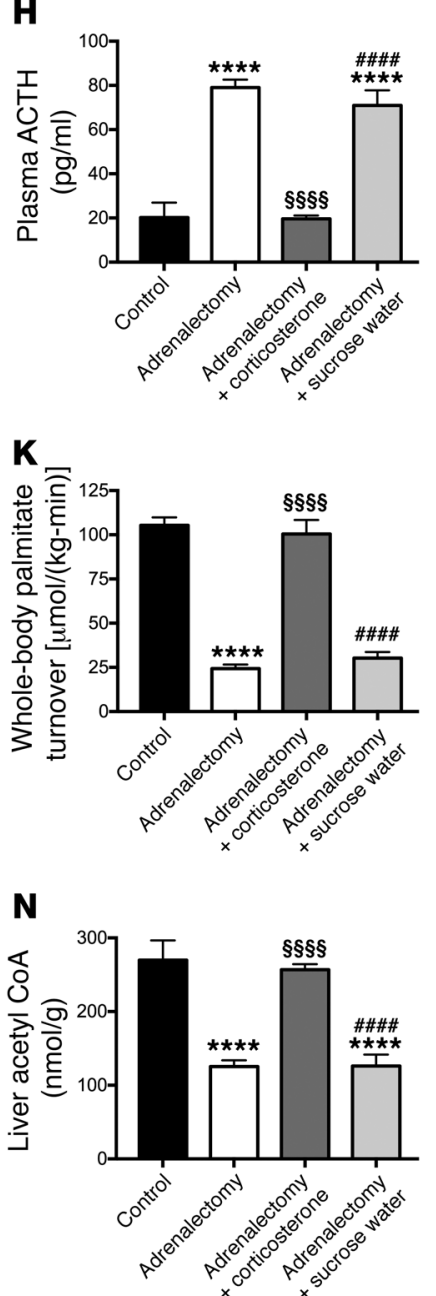

C
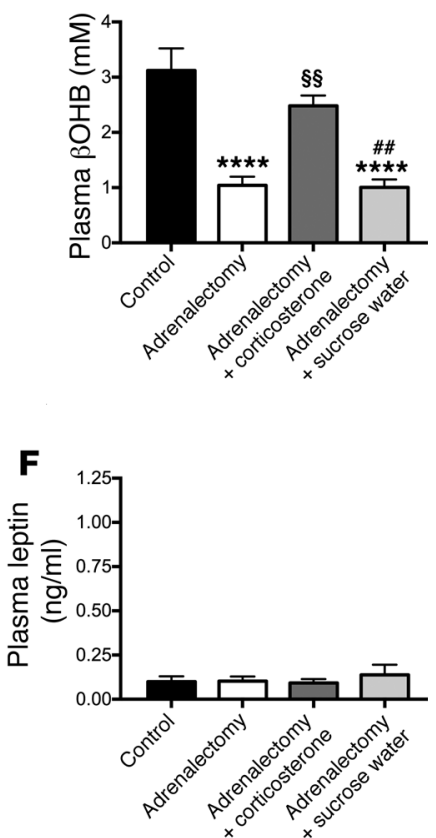

I

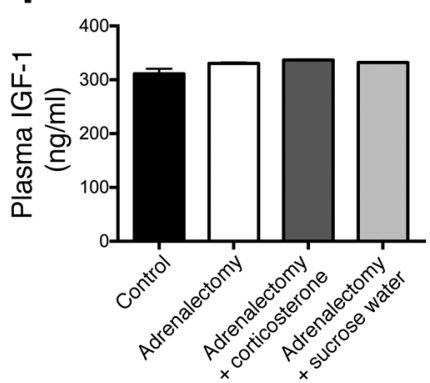

$\mathbf{L}$
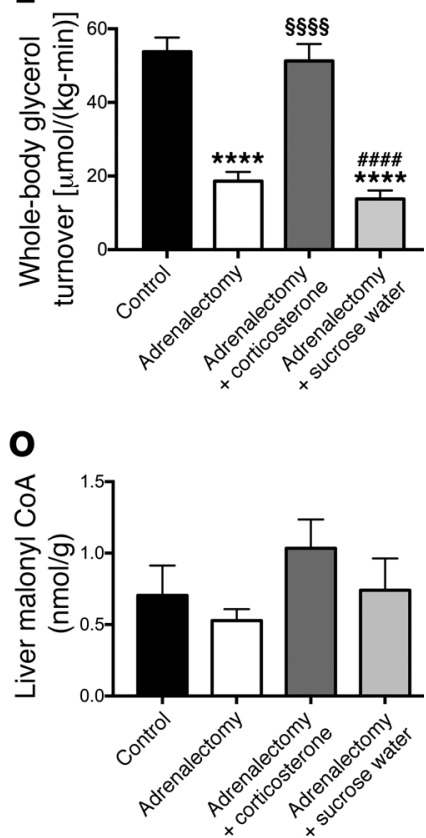

Figure 4. Hypercorticosteronemia drives DKA through increased lipolysis. (A-C) Plasma glucose, NEFA, and $\beta O H B$ concentrations in adrenalectomized T1D rats. In all panels, unless indicated otherwise, all measurements were made at the 4-hour time point. (B) ${ }^{*} P<0.05$, ${ }^{* *} P<0.01,{ }^{* * *} P<0.001$ ${ }^{* * * *} P<0.0001$ between the groups indicated; ${ }^{\S \S} P<0.01$ vs. adrenalectomy plus corticosterone at time zero. (D) Anion gap. (E-I) Plasma insulin, leptin, corticosterone, $\mathrm{ACTH}$, and IGF-1 concentrations. (J) Whole-body glucose turnover. (K-M) Whole-body palmitate, glycerol, and $\beta \mathrm{OHB}$ turnover. (N and $\mathbf{0}$ ) Liver acetyl and malonyl CoA concentrations. In all panels, data are the mean \pm SEM of $n=8$ (control), $n=11$ (adrenalectomy), $n=6$ (adrenalectomy plus corticosterone), or $n=6$ (adrenalectomy plus sucrose water) per group. Unless otherwise specified, ${ }^{*} P<0.05,{ }^{* *} P<0.01,{ }^{* *} P<$ $0.001,{ }^{* * *} P<0.0001$ vs. controls; ${ }^{\S} P<0.05,{ }^{\S \S} P<0.01,{ }^{\S \S \S} P<$ $0.001,{ }^{\$ \$ \$ \$} P<0.0001$ vs. adrenalectomized rats; $\# P<0.01$, \#\#\# $P$ $<0.001$, \#\#\#\# $P<0.0001$ vs. adrenalectomy plus corticosteronetreated rats. Data were compared using ANOVA with Bonferroni's multiple comparisons test. 
A
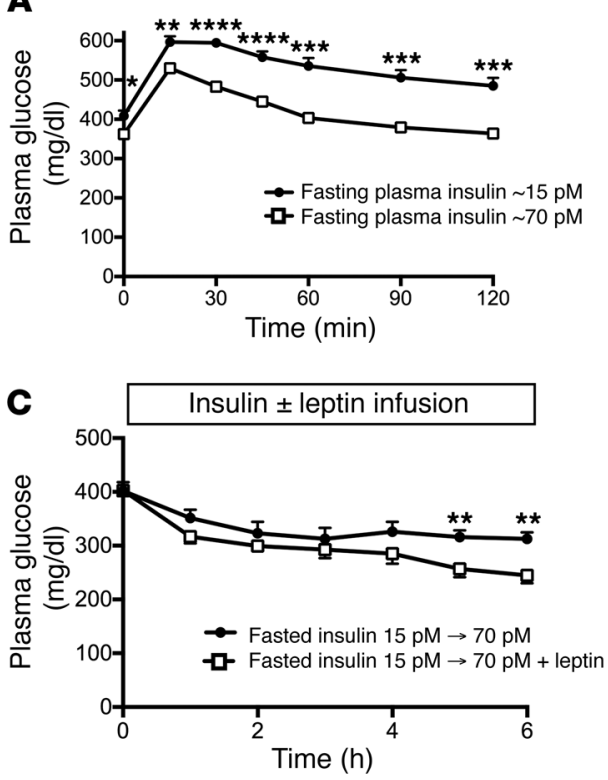

E

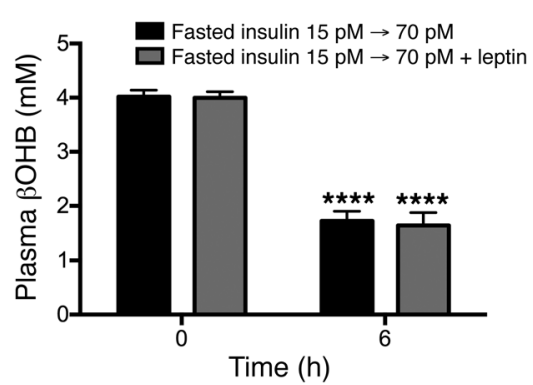

G

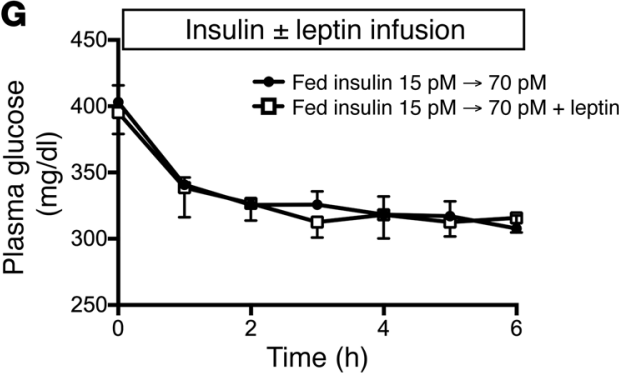

I

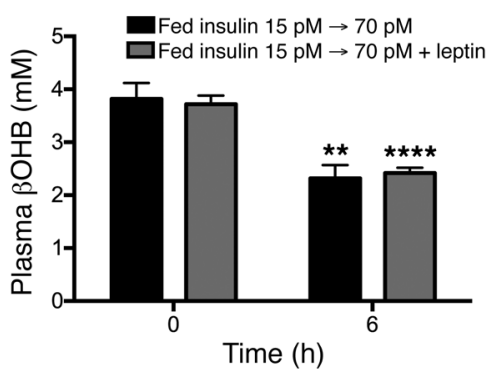

B

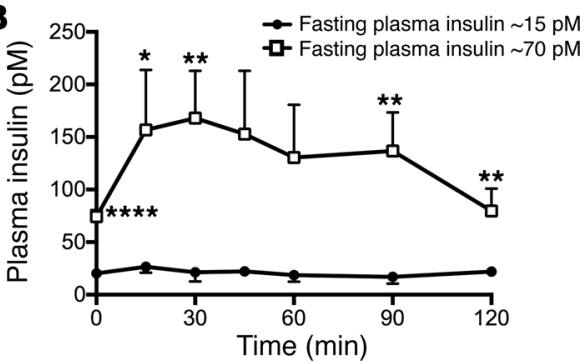

D

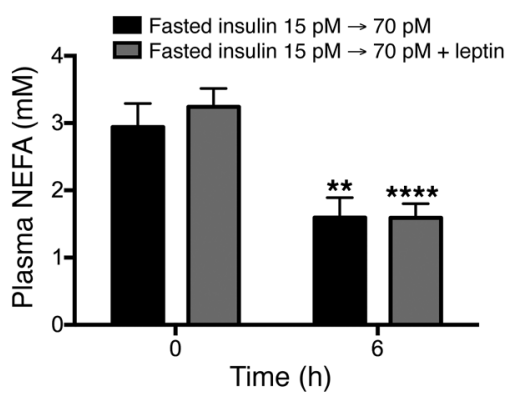

$\mathbf{F}$

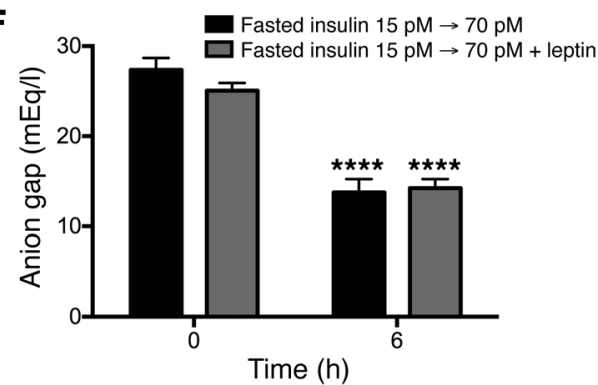

H

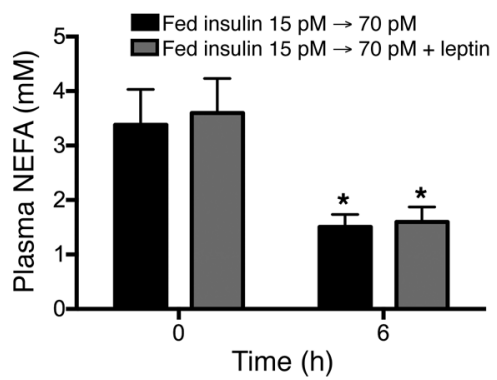

J

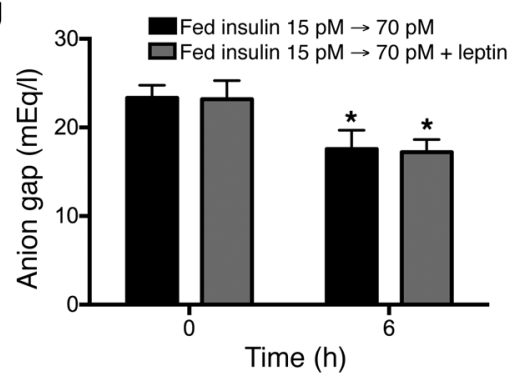

Figure 5. Insulin and feeding abrogate leptin's effects to lower plasma glucose. (A and $\mathbf{B}$ ) Plasma glucose and insulin during a glucose tolerance test in T1D rats with fasting plasma insulin of approximately $15 \mathrm{pM}$ as compared with those with fasting plasma insulin of approximately $70 \mathrm{pM}$. (C-F) Plasma glucose, NEFA, $\beta O H B$, and anion gap before and after an infusion of insulin to achieve plasma concentrations of approximately $70 \mathrm{pM}$. (G-J) Plasma glucose, NEFA, $\beta \mathrm{OHB}$, and anion gap in fed rats with food withdrawn at the start of the study, before and after an infusion of insulin to achieve plasma concentrations of approximately 70 pM. (A-C, and G) ${ }^{*} P<0.05$, ${ }^{*} P<0.01$, ${ }^{* * *} P<0.001,{ }^{* * *} P<0.0001$ between groups by 2-tailed unpaired Student's $t$ test. (D-F and $\mathbf{H}-\mathbf{J}){ }^{*} P<0.05$, ${ }^{* *} P<0.01,{ }^{* * *} P<0.0001$ vs. the same group at time zero by 2 -tailed paired Student's $t$ test. Data are the mean \pm SEM of $n=6$ per group (panels A-F) or $n=10$ (fed, insulin $15 \mathrm{pM}$ to 70 $\mathrm{pM}$ ) and $n=11$ (fed, insulin $15 \mathrm{pM}$ to 70 $\mathrm{pM}+$ leptin) per group (panels $\mathbf{G}-\mathbf{J}$ ). only modestly reducing plasma glucose in this setting, despite a $40 \%$ reduction in plasma corticosterone in rats infused with insulin (Figure 5, C-F, and Supplemental Figure 3, C-E). Rates of HGP, lipolysis, and $\beta \mathrm{OHB}$ turnover as well as liver acetyl and malonyl CoA concentrations were unchanged with leptin treatment (Supplemental Figure 3, F-K), but with the exception of malonyl CoA content, each parameter was lower than that measured in rats that were in DKA and had plasma insulin concentrations of approximately $15 \mathrm{pM}$ (Figure $1 \mathrm{H}$ and Figure 2, D-H).

Next, we assessed the impact of acute leptin infusion on glycemia in fed (3-hour food withdrawn) rats infused with insulin to achieve plasma insulin concentrations of approximately $70 \mathrm{pM}$ 
A
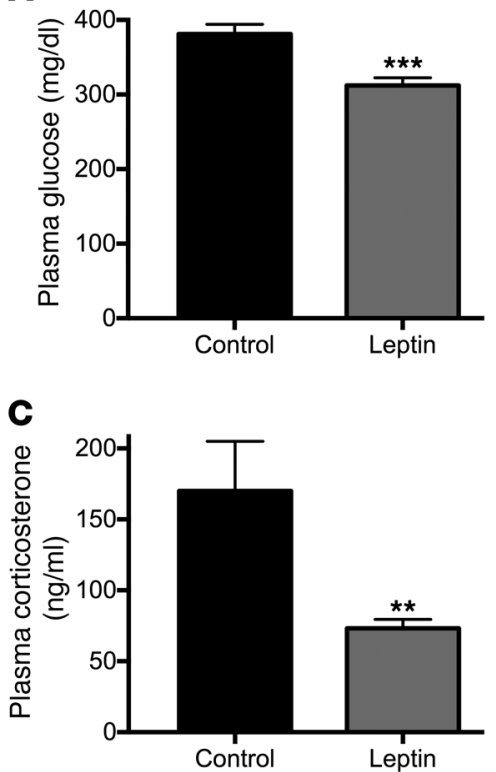

E

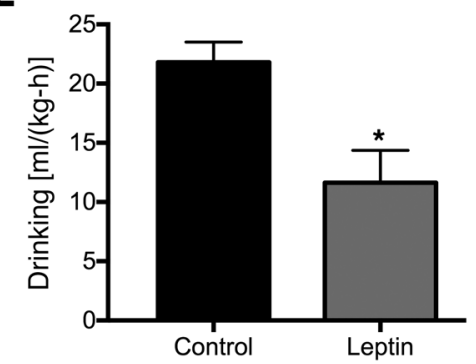

G

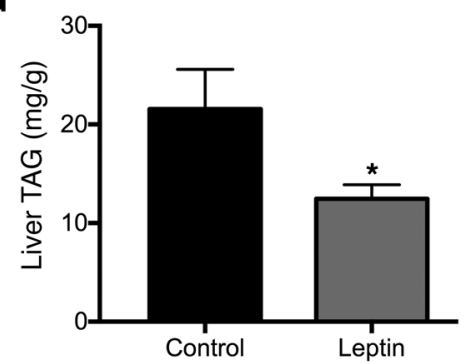

I

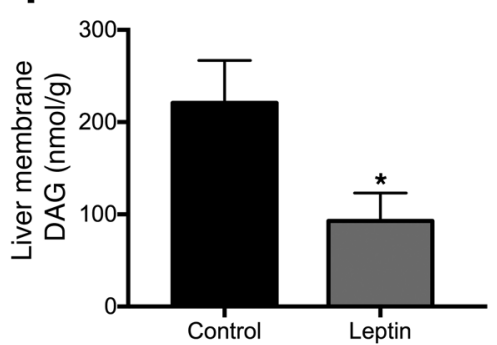

B

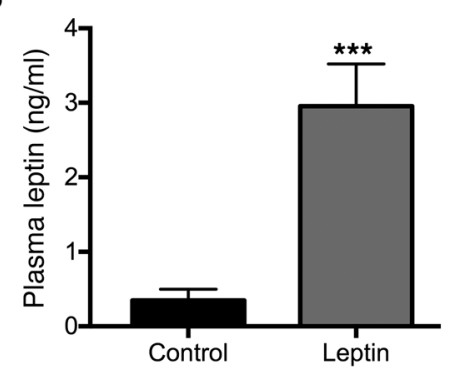

D

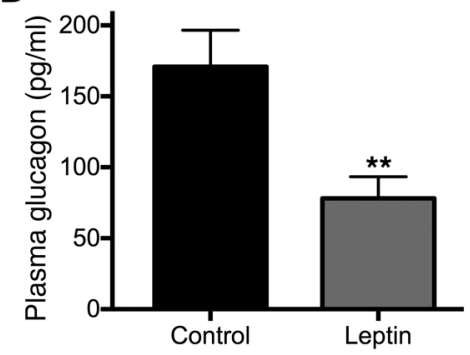

$\boldsymbol{F}$

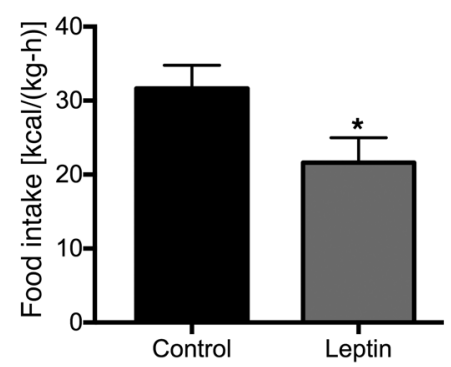

H

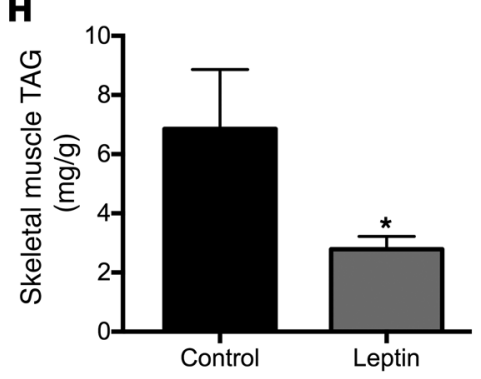

J

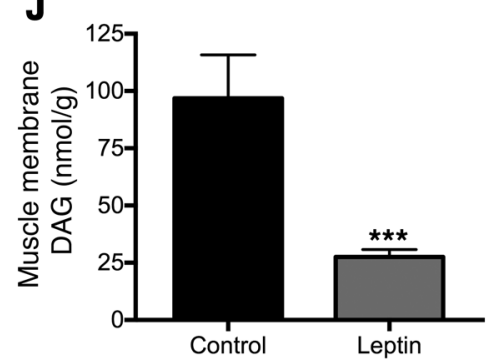

Figure 6. The chronic effect of leptin to suppress hyperglycemia in T1D mice is pleotropic. (A and B) Plasma glucose and leptin concentrations in mice infused for 2 weeks with leptin or saline (control). (C and $\mathbf{D})$ Plasma corticosterone and glucagon. (E) Water drinking. (F) Food intake. (G and $\mathbf{H}$ ) Liver and skeletal muscle TAG concentrations. (A-H) Data are the mean \pm SEM of $n=10$ (control) or $n=12$ (leptin-treated) per group. (I and J) Liver and skeletal muscle DAG concentrations, $n=8$ (control) or $n=10$ (leptin-treated) per group. ${ }^{*} P<0.05,{ }^{* *} P<0.01$, ${ }^{* *} P<0.001$ by 2 -tailed unpaired Student's $t$ test.

(Supplemental Figure 4, A and B). Similar to what we observed in the fasted animals, despite leptin's effect to suppress plasma corticosterone concentrations in these fed rats, leptin had no additional effect on plasma glucose, NEFA or $\beta O H B$ concentrations, anion gap, whole-body glucose, fatty acid, glycerol, or $\beta \mathrm{OHB}$ turnover or hepatic acetyl or malonyl CoA content, beyond the effect of insulin to suppress those parameters (Figure 5, G-J and Supplemental Figure 4, C-I).

Chronic leptin treatment reduces hyperglycemia in a mouse model of T1D. In order to examine the mechanisms by which chronic leptin replacement therapy reverses hyperglycemia in nonketoacidotic diabetic rodents, we treated STZ-induced T1D mice with a 2-week subcutaneous infusion of either leptin or saline by ALZET pump. This intervention increased plasma leptin concentrations 6-fold and was associated with a $75 \mathrm{mg} / \mathrm{dl}$ reduction in 6-hour-fasted plasma glucose concentrations despite unchanged insulinopenia (Figure 6, A and B, and Supplemental Figure 5A). Chronic leptin treatment was also associated with a $30 \%$ reduction in plasma NEFA concentrations and 50\% reductions in plasma corticosterone and glucagon concentrations (Figure 6, C and D, and Supplemental Figure $5 \mathrm{~B})$. Lower plasma glucose concentrations were associated with a $50 \%$ reduction in water drinking in leptin-treated mice, while their increase in plasma leptin and reductions in corticosterone and glucagon were also associated with a $30 \%$ suppression of food intake. Chronic leptin treatment had no effect on energy expenditure, oxygen consumption, or carbon dioxide production (Figure 6, E and F, and Supplemental Figure $5, C-G)$. However, the reductions in food intake in leptin-infused rats resulted in 50\%-70\% lower triacylglycerol (TAG) and diacylglycerol (DAG) content in liver and skeletal muscle, without any difference in liver or muscle ceramide concentrations (Figure 6, G-J, and Supplemental Figure 5, $\mathrm{H}$ and I).

\section{Discussion}

It is well established that leptin replacement therapy lowers plasma glucose concentrations in insulinopenic rodent models of type 1 diabetes $(1-8,10,13)$. The mechanism or mechanisms by which leptin mediates these acute and/or chronic effects remain unclear. We recently found that acute leptin replacement therapy reduces plasma glucose concentrations by suppressing plasma corticosterone concentrations, resulting in lower rates of lipolysis, which in turn leads to reductions in hepatic acetyl CoA content and HGP in 3 rat models of poorly controlled diabetes (12). However, Morton et al. found that leptin as well as glucocorticoid receptor blockade had no effect on plasma glucose concentrations in a STZ-treated rat model of T1D and that adrenalectomized T1D rats did not exhibit plasma glucose concentrations lower than those of nonadrenalectomized T1D rats (13). One possible explanation for the 
different results could be differences in the diabetic rat model. In contrast to our T1D rat model, which was severely insulinopenic $(\sim 15 \mathrm{pM})$, and in DKA, the Morton et al.STZ-induced rat model had basal insulin concentrations that were 4 - to 5-fold higher ( 70pM) than those in our T1D rat model. Furthermore, in contrast to our acute studies, which were all performed following a 12-hour overnight fast, many of the studies performed by Morton et al. were chronic studies in fed rats, or were performed in rats that had food removed for only 3 hours. In order to examine whether these differences in the diabetic rat models could account for the disparate findings as well as to further elucidate the insulin-independent mechanism by which leptin acutely reverses DKA, we performed a series of experiments of acute leptin replacement therapy under several conditions to replicate the conditions used in both studies.

First, in order to examine the mechanism by which leptin replacement therapy acutely reverses DKA in an insulin-independent manner, we examined the effects of leptin replacement therapy on rates of lipolysis, HGP, hepatic ketogenesis, hepatic acetyl CoA content, and malonyl CoA content in a rat model of STZinduced DKA with plasma insulin concentrations of approximately $15 \mathrm{pM}$. Consistent with our previous study (12) and that of Morton et al. (13), STZ-induced diabetic rats exhibited hyperglycemia, hypoleptinemia, and hypercorticosteronemia compared with normal rats not treated with STZ, all of which were reversed with an acute leptin infusion. Leptin's acute effect to lower plasma glucose concentrations could be attributed to a greater than $40 \%$ reduction in rates of HGP (Figure $1 \mathrm{H}$ ). To understand the mechanism for this effect, we measured plasma glucagon, epinephrine, norepinephrine, and growth hormone concentrations as well as IGF-1 and found that acute leptin replacement therapy did not change concentrations of any of these hormones, in contrast to its effect of reducing plasma corticosterone concentrations by $50 \%$. However, replacing corticosterone in leptin-infused rats caused hyperglycemia, which could be attributed to a 2 -fold increase in rates of endogenous glucose production. These changes were associated with a doubling in rates of lipolysis and hepatic acetyl CoA content, but no change in malonyl CoA content (Figure 1 and Figure 2). Taken together, these data demonstrate that leptin-induced suppression of plasma corticosterone concentration is necessary to mediate leptin's acute insulin-independent effects to decrease rates of HGP and reverse hyperglycemia in DKA.

To determine whether increased rates of lipolysis promote increased rates of HGP and ketogenesis in DKA, we next treated leptin- and corticosterone-infused rats with atglistatin, a small molecule inhibitor of ATGL. As expected, ATGL inhibition led to marked decreases in lipolysis, as reflected by acute reductions in rates of both palmitate and glycerol turnover. Reduced rates of lipolysis in turn resulted in an approximately 50\% reduction in rates of HGP, ketogenesis, and hepatic acetyl-CoA content, thereby demonstrating that suppression of lipolysis is sufficient to reverse DKA. Taken together, these data demonstrate that leptin's acute insulin-independent effect to reverse DKA is mediated by leptin-induced suppression of hypercorticosteronemia, resulting in reductions in lipolysis.

Surprisingly, despite reversal of DKA, neither leptin nor ATGL treatment resulted in any changes in hepatic malonyl CoA content (nondiabetic controls, $0.51 \pm 0.08 \mathrm{nmol} / \mathrm{g}$ ) (Supplemental Figure
1I), thus dissociating reversal of DKA from changes in this key regulator of CPT-I activity and hepatic fatty acid oxidation (16). We hypothesized that increased hepatic acetyl CoA, derived from increased lipolysis and fatty acid delivery to the liver, was the main driver of increased hepatic gluconeogenesis through activation of pyruvate carboxylase in the setting of DKA (12). Consistent with this hypothesis, leptin-treated T1D rats infused with acetate to match hepatic acetyl CoA concentrations to those of untreated T1D controls were hyperglycemic and ketotic without any changes in hepatic malonyl CoA content (Figure 3). Collectively, these data directly implicate increases in hepatic acetyl CoA as a major driver of increased hepatic gluconeogenesis and demonstrate that increased hepatic ketogenesis in DKA is driven mostly by acute increases in fatty acid delivery to the liver and not by reductions in hepatic malonyl CoA content.

Next, we sought to understand why Morton et al. did not observe any chronic differences in plasma glucose concentrations in adrenalectomized STZ-induced diabetic rats (13). As expected, we found that adrenalectomized T1D rats had almost undetectable plasma corticosterone concentrations and higher plasma ACTH concentrations than sham-operated T1D controls without any difference in plasma glucose concentrations when given drinking water containing sodium chloride, sucrose, and corticosterone; however, within 1 hour of replacing this water mixture with water containing only sodium chloride, plasma glucose concentrations rapidly decreased by $75 \mathrm{mg} / \mathrm{dl}$ and continued to fall to approximately $125 \mathrm{mg} / \mathrm{dl}$ within 4 hours of removal of the sucrose/ corticosterone water. This striking reduction of plasma glucose concentrations could be explained by reductions in whole-body glucose turnover driven by lower rates of lipolysis and hepatic acetyl CoA content and was accompanied by a reversal of DKA and suppression of $\beta \mathrm{OHB}$ turnover (Figure 4). However, infusion of corticosterone to match plasma concentrations in sham-operated T1D controls abrogated each of these effects of adrenalectomy. Taken together, these data demonstrate that hypercorticosteronemia is necessary for acute ketoacidosis and that insulinopenia alone is necessary but not sufficient to induce DKA.

Our data also suggest that the inability of Morton et al. to observe any glucose-lowering effect of adrenalectomy is likely the result of administering sucrose-containing water to their polydipsic diabetic adrenalectomized rats. In the aforementioned study, the authors state that variations in the amount of sucrose water intake did not affect glycemia in diabetic animals (13). However, Morton et al. did not assess plasma glucose concentrations in adrenalectomized diabetic rats in the absence of sucrose water. To examine this possibility, we studied adrenalectomized T1D rats maintained on water containing $1 \%$ sucrose and sodium chloride. Here, we found that, consistent with the findings of Morton et al. (13), providing diabetic rats with sucrose water increased plasma glucose concentrations by more than $200 \mathrm{mg} / \mathrm{dl}$ relative to adrenalectomized diabetic rats given water containing only sodium chloride. Thus, in fed adrenalectomized diabetic rats, such as those given sucrose-containing water, suppression of hypercorticosteronemia is insufficient to normalize plasma glucose concentrations.

In addition to the striking effect of sucrose in the drinking water to abrogate the effect of glucocorticoid deficiency to decrease plasma glucose concentrations in diabetic rats, we also hypothesized 
that the 5-fold higher plasma insulin concentrations $(\sim 70 \mathrm{pM})$ in the STZ-treated diabetic rat model studied by Morton et al. may have also contributed to their inability to detect any effects of acute leptin treatment on plasma glucose concentrations (13). T1D rats with fasting plasma insulin concentrations of approximately 70 pM retain the ability to secrete substantial amounts of insulin in response to a glucose challenge, unlike insulinopenic rats with fasting plasma insulin concentrations of approximately $15 \mathrm{pM}$, which do not increase following a glucose challenge, demonstrating the absence of any pancreatic $\beta$ cell reserve in our diabetic rodent model. Importantly, rats with fasting plasma insulin concentrations of approximately $70 \mathrm{pM}$ are not in DKA, as indicated by their lower $\beta \mathrm{OHB}$ concentrations and normal anion gap. To further clarify this point, we infused insulin to increase plasma insulin concentrations from approximately 15 to approximately $70 \mathrm{pM}$ in overnightfasted T1D rats. We found that this intervention acutely suppressed lipolysis, hepatic acetyl CoA, HGP, and plasma glucose concentrations. In the setting of approximately $70 \mathrm{pM}$ insulin, leptin had a very modest effect on plasma glucose and corticosterone and no additional effect on lipolysis, similar to Morton et al.'s data (13) and demonstrating that the effect of insulin to suppress hypercorticosteronemia, lipolysis, and HGP nullifies any additional effect of leptin to further suppress these parameters. Similarly, and consistent with our data in adrenalectomized rats given sucrose water, leptin replacement therapy had no additional effect of lowering plasma glucose, glucose turnover, lipolysis, ketosis, or hepatic acetyl CoA in fed rats, again consistent with the findings of Morton et al. (13) and suggesting that the chronic effects of leptin in the postprandial state are minimally or not at all reliant upon suppression of hypothalamic-pituitary-adrenal (HPA) axis activity. These data demonstrate that insulin - even insulin concentrations insufficient to achieve euglycemia - will nullify the effects of hypercorticosteronemia to stimulate lipolysis, HGP, and hyperglycemia and likely explain the inability of Morton et al. to observe an acute effect of leptin to reduce hyperglycemia in their diabetic rat studies. In addition, and in contrast to this and our previous studies $(12,17)$, Morton et al. did not assess metabolite turnover or liver metabolite concentrations, so it is impossible to determine whether leptin had any effect on rates of lipolysis, rates of HGP, rates of hepatic ketogenesis, or hepatic acetyl CoA content in their studies (13). Thus, the lack of an effect of leptin in these animals may represent an inability of leptin to reverse postprandial hyperglycemia, which is mostly an insulin-dependent condition, and/or to suppress lipolysis beyond the extent to which it is already suppressed by insulin concentrations of approximately $70 \mathrm{pM}$ (17).

The results from our studies and those of Morton et al. clearly demonstrate that leptin has little to no acute effect on plasma glucose in diabetic rats that have plasma insulin concentrations of $70 \mathrm{pM}$ or more and are not in DKA. Thus, the acute mechanism of action of leptin to reverse DKA by suppression of HPA axis activity is unlikely to explain the chronic glucose-lowering effect of leptin observed by other groups in diabetic rodents that are not completely insulin deficient and not in DKA $(1-3,7)$.

To better understand the mechanism or mechanisms for the chronic glucose-lowering effects of leptin, we treated a STZinduced mouse model of poorly controlled T1D with leptin by subcutaneous ALZET pump for 2 weeks. This intervention low- ered plasma glucose concentrations in 6-hour-food-withdrawn animals without affecting plasma insulin concentrations. The lower plasma glucose concentrations exhibited by the leptin-treated mice were associated with reductions in both plasma corticosterone and glucagon, consistent with previous studies $(8,12)$. Chronic leptin treatment lowered both water drinking, consistent with lower plasma glucose concentrations throughout the day, and food intake, similar to its effect in humans with poorly controlled diabetes due to lipodystrophy (18), but did not affect activity or energy expenditure. As ectopic lipid content and, more importantly, intracellular DAG content have been strongly implicated in causing insulin resistance in liver and skeletal muscle, the lower plasma glucose concentrations in chronic leptin-infused mice can be attributed, at least in part, to lower liver and muscle DAG content and reversal of insulin resistance in these tissues (19-21). In contrast these glucose lowering effects of chronic leptin treatment occurred independently of any changes in liver and muscle ceramide content. Interestingly, a growing body of literature suggests that leptin may actually promote lipolysis by increasing catecholamine concentrations on a chronic basis in normal rodents, as reviewed recently by Ruud and Brüning (22). However, the fact that plasma NEFA concentrations actually dropped in our chronic leptin-treated T1D mice suggests that, in models of poorly controlled diabetes, the effect of leptin in suppressing HPA axis activity in hypercorticosteronemic diabetic rodents trumps its potential to promote catecholamine release and would be consistent with the idea that suppression of HPA axis activity may not be central to leptin's chronic effect to normalize glycemia in rodents with T1D. Taken together, our data combined with results from previous studies suggest that the chronic effects of leptin to reduce both fasting and postprandial hyperglycemia in leptin-deficient diabetic rodents not in DKA are likely pleotropic and may have minimal dependence on suppression of the HPA axis. Based on these results, we hypothesize that the chronic effect of leptin can be attributed at least in part to its ability to (a) decrease hyperphagia, resulting in reductions in postprandial hyperglycemia; (b) lower ectopic lipid (DAG) content in liver and skeletal muscle, resulting in reversal of insulin resistance in these organs $(7,18)$; (c) reduce plasma corticosterone concentrations; and (d) reduce plasma glucagon concentrations $(1,8,23-25)$. All of these chronic changes will also result in reductions in glucose toxicity, which will further improve insulin responsiveness in liver and skeletal muscle and enhance pancreatic $\beta$ cell activity $(26,27)$.

In summary, we examined the acute insulin-independent effects of leptin replacement therapy in an STZ-induced rat model of DKA and found that (a) hypercorticosteronemia is necessary to cause increased rates of lipolysis, HGP, and hepatic ketogenesis in DKA; (b) leptin's acute insulin-independent ability to reverse DKA is mediated by suppression of the HPA axis, resulting in reductions in hypercorticosteronemia; (c) relatively low concentrations of insulin $(\sim 70 \mathrm{pM})$ are sufficient to suppress lipolysis, hepatic acetyl CoA, and HGP and abrogate the acute effects of leptin to reduce plasma glucose concentrations in a rat model of poorly controlled T1D; (d) reversal of DKA by leptin and insulin is mostly driven by reduction in rates of lipolysis and independent of any changes in hepatic malonyl CoA content; and (e) surprisingly, as demonstrated in the adrenalectomized T1D rats, insulinopenia per se is necessary but not 
sufficient to cause DKA. These data demonstrate that suppression of hypercorticosteronemia, lipolysis, and hepatic acetyl CoA mediates leptin's acute insulin-independent effect to reverse DKA.

In contrast, we found that the chronic effects of leptin to reduce both fasting and postprandial hyperglycemia in leptindeficient diabetic rodents not in DKA are likely pleotropic and can be attributed at least in part to leptin's ability to decrease hyperphagia, resulting in reductions in postprandial hyperglycemia and ectopic lipid content in liver and skeletal muscle that in turn result in reversal of insulin resistance in these organs as well as chronic reductions in plasma glucagon and corticosterone concentrations. Taken together, these studies demonstrate marked differences in the acute insulin-independent effects by which leptin reverses fasting hyperglycemia and ketoacidosis in a rat model of DKA versus the chronic effects by which leptin reverses hyperglycemia in a less insulinopenic, non-DKA rodent model of T1D. Furthermore, these results resolve the discordant findings between our studies examining the glucose-lowering effects of leptin to reverse DKA in the absence of insulin (12) and studies examining the chronic effects of leptin to reduced hyperglycemia in diabetic rodents that still retain some $\beta$ cell reserve (13).

\section{Methods}

Animals. Male Sprague-Dawley rats weighing approximately $350 \mathrm{~g}$ were ordered from Charles River Laboratories and were allowed to acclimate for 1 week before undergoing surgery under general isoflurane anesthesia to place polyethylene catheters in the jugular vein with the tip advanced to the right atrium and in the carotid artery (PE90 and PE50 tubing, respectively; Instech Laboratories). Adrenalectomized rats were obtained from Harlan and were given ad libitum access to water containing $2 \%$ sucrose, $0.9 \%$ sodium chloride, and $25 \mu \mathrm{g} / \mathrm{ml}$ corticosterone (all from Sigma-Aldrich). In surgery, the rats were given an injection of $1 \mathrm{ml}$ of this solution, and the concentrations of these agents in the water were doubled for 48 hours after surgery to provide stress dose coverage. Adrenalectomized rats had their food withdrawn overnight prior to study, but they continued to receive sucrose and corticosterone in their water until the morning of the study $(8 \mathrm{am})$, when they had their sucrose-corticosterone-saline water replaced with water containing $0.9 \%$ sodium chloride only or $1 \%$ sucrose and $0.9 \%$ sodium chloride, as indicated in Figure 4 and Supplemental Figure 3 legends. They were observed continuously, and plasma glucose was measured hourly after removal of the sucrose and/or corticosterone from their water.

In all rats, diabetes was induced by i.p. injection of STZ (Sigma-Aldrich, $65 \mathrm{mg} / \mathrm{kg}$ ) 24 hours prior to the start of the infusion studies. Unless otherwise specified, in which case food was withdrawn 3 hours prior to sacrifice (beginning at $8 \mathrm{am}$ ), rats were fasted overnight (beginning at 6pm) the night before the study, and the next morning, those with plasma glucose concentrations of greater than $300 \mathrm{mg} / \mathrm{dl}$ were studied, beginning at $8 \mathrm{am}$. The venous catheter was used for blood draws (after 1 hour of rest following connection of their catheters to reduce any impact of stress on plasma glucose concentrations), while the arterial catheter was used for infusion of substrates and hormones as detailed in the following section. Unless otherwise specified, rats were later excluded from the study if they had plasma insulin concentrations of $20 \mathrm{pM}$ or more at time zero of the infusion or anion gap of $20 \mathrm{mEq} / \mathrm{l}$ or less.
To study rats with modest insulinopenia, a lower dose $(40 \mathrm{mg} /$ $\mathrm{kg}$ ) of STZ was used to induce diabetes using the same timeline as described above, and hyperglycemic (fasting plasma glucose $\geq 300$ $\mathrm{mg} / \mathrm{dl}$ ) rats were later divided into groups with fasting plasma insulin of approximately $15 \mathrm{pM}(\leq 20 \mathrm{pM})$ or approximately $70 \mathrm{pM}(>60$ $\mathrm{pM})$. They underwent a glucose tolerance test with i.v. administration of $1 \mathrm{~g} / \mathrm{kg}$ dextrose at time zero. Plasma was obtained through a venous catheter at the time points indicated, and plasma glucose and insulin were measured as described above.

Mice were obtained from The Jackson Laboratory at 8 weeks of age and allowed to acclimate for 1 week before they were treated with an i.p. injection of STZ (125 mg/ kg). Three days later, those with fed blood glucose concentrations of more than $300 \mathrm{mg} / \mathrm{dl}$ were randomized to receive placement of an ALZET 1004 pump containing either leptin or saline, which infused leptin at a rate of $0.624 \mu \mathrm{g} / \mathrm{h}$. During the second week of the leptin infusion, the mice underwent CLAMS metabolic cage analysis to measure daily eating, drinking, activity, oxygen consumption, carbon dioxide production, respiratory exchange ratio, and energy expenditure. After 2 weeks of leptin or saline infusion, following a 6-hour-food-withdrawal period ( 8 am to $2 \mathrm{pm}$ ), mice were sacrificed under isoflurane anesthesia. Blood was obtained from the aorta using a syringe, and liver and skeletal muscle (gastrocnemius + soleus) were isolated.

Tracer infusions. For the last 2 hours of the infusion studies, all rats were infused continuously with 4 tracers concurrently: $[1,2,3,4,5,6,6-$ ${ }^{2} \mathrm{H}_{7}$ ] glucose (0.1 mg/[kg-min], Cambridge Isotopes), $\left[{ }^{13} \mathrm{C}_{4}\right] \beta \mathrm{OHB}(0.1$ $\mathrm{mg} /\left[\mathrm{kg}\right.$-min], Cambridge Isotopes), $\left[{ }^{13} \mathrm{C}_{16}\right]$ potassium palmitate $(0.2$ $\mathrm{mg} /\left[\mathrm{kg}\right.$-min], Cambridge Isotopes), and $\left[1,1,2,3,3{ }^{-2} \mathrm{H}_{5}\right]$ glycerol (0.1 $\mathrm{mg} /[\mathrm{kg}$-min], Cambridge Isotopes) for 120 minutes. Tracers were infused through the arterial catheter and blood sampled through the venous, following the optimal method described for tracer infusion studies (28-30). The blood sample taken at the last time point, after 120 minutes of tracer infusion, was used to measure steady-state plasma enrichment of each metabolite of interest, from which the turnover rate of the metabolite could be calculated.

Acute leptin and corticosterone infusions. T1D rats were randomized using a random number generator to receive control (saline) or leptin with or without corticosterone infusions. Rats were treated with a 6-hour continuous infusion of leptin $(0.125 \mathrm{mg} / \mathrm{kg}$ total) or an equivalent volume of saline $(0.1875 \mathrm{ml})$ starting at 8 am and concluding at $2 \mathrm{pm}$. A subgroup of leptin-treated rats were coinfused with corticosterone $(12.5 \mathrm{mg} / \mathrm{kg}$ total) continuously during the 6-hour leptin infusion. In this and all other leptin infusion studies, blood (200 $\mu$ l whole blood) was drawn through the venous catheter hourly into a heparin-lithium-aprotinin-coated tube and centrifuged immediately; plasma was isolated and stored at $-80^{\circ} \mathrm{C}$, awaiting further analysis. The same protocol was used to treat adrenalectomized rats with corticosterone for their 4-hour period of study after removal of their sucrose and corticosterone water. In all cases, substrates and hormones were infused through the arterial catheter and blood was drawn through the venous catheter to avoid any contamination of the blood in which we planned to measure concentrations of the substance or substances being infused.

Insulin infusions. Groups of fed (3-hour food withdrawn) and overnight-fasted rats were infused with a primed-continuous infusion of insulin ( $4 \mathrm{mU} / \mathrm{kg}$ prime, $0.4 \mathrm{mU} /[\mathrm{kg}-\mathrm{min}]$ continuous infusion) through the duration of a 6-hour infusion of leptin or saline as described above. 
Acetate infusions. Separate groups of T1D rats were infused with leptin as described above or leptin plus acetate $[75 \mu \mathrm{mol} /(\mathrm{kg}-\mathrm{min})]$ for the 6-hour duration of the leptin infusion ( 8 am to $2 \mathrm{pm}$ ) or for the last 2 hours of the leptin infusion.

Atglistatin treatment. Fifteen minutes prior to beginning a 6-hour infusion of leptin plus corticosterone, as described above, we injected atglistatin $(200 \mu \mathrm{mol} / \mathrm{kg}$, Xcess Biosciences $)$ in $500 \mu \mathrm{l}$ saline. In order to solubilize the drug, we added $50 \mathrm{mg}$ Cremophor (Sigma-Aldrich) and $100 \mu \mathrm{l} 1 \mathrm{M} \mathrm{HCl}$, adjusted the $\mathrm{pH}$ to approximately 7.0 with dropwise addition of Tris base (Sigma-Aldrich), vortexed, and checked the $\mathrm{pH}$ with $\mathrm{pH}$ strips after each addition of base. We then sonicated the solution for 10 minutes in a $37^{\circ} \mathrm{C}$ water bath. The solution was injected i.p. immediately after solubilizing and cooling to $25^{\circ} \mathrm{C}$.

Flux analysis. Plasma samples were deproteinized and derivatized to measure $\left[{ }^{2} \mathrm{H}\right]$ glucose and glycerol enrichment as follows. $100 \mu \mathrm{l}$ methanol (Sigma-Aldrich) was added to $20 \mu \mathrm{l}$ plasma,which was centrifuged, and the resulting supernatant was dried under $\mathrm{N}_{2}$ gas. The sample was then derivatized in $75 \mu \mathrm{l}$ acetic anhydride/pyridine (1:1, both from Sigma-Aldrich), heated to $65^{\circ} \mathrm{C}$ for 20 minutes, and after cooling to room temperature and adding $25 \mu \mathrm{l}$ methanol, $\mathrm{m}+7\left[{ }^{2} \mathrm{H}\right]$ glucose enrichment was assessed by gas chromatography-mass spectrometry (GC/MS) (chemical ionization [CI] mode, mass to charge ratio $[\mathrm{m} / \mathrm{z}] 331[\mathrm{~m}+0]$ and $338[\mathrm{~m}+7]) .\left[{ }^{2} \mathrm{H}\right]$ glycerol enrichment was subsequently assessed in the same samples, also using GC/MS (electron ionization [EI] mode, $\mathrm{m} / \mathrm{z} 145[\mathrm{~m}+0]$ and $148[\mathrm{~m}+5])$.

$\beta \mathrm{OHB}$ enrichment was also measured by GC/MS. Samples $(25 \mu \mathrm{l}$ plasma) were dried under $\mathrm{N}_{2}$ gas and derivatized with $75 \mu \mathrm{l} N$-butanol $4 \mathrm{~N}$ $\mathrm{HCl}$ (both from Sigma-Aldrich), then heated for 30 minutes at $60^{\circ} \mathrm{C}$ and dried overnight in a $60^{\circ} \mathrm{C}$ vacuum oven. The following day, the samples were reacted with $100 \mu \mathrm{l}$ of trifluoroacetic acid (Thermo Fisher Scientific) and methylene chloride (Sigma-Aldrich) (1:7). $\left.{ }^{13} \mathrm{C}_{4}\right] \beta \mathrm{OHB}$ enrichment was measured by GC/MS (EI mode, $\mathrm{m} / \mathrm{z} 257$ [m+0] and 261 [m+4]).

Palmitate enrichment was measured by GC/MS. Plasma samples $(25 \mu \mathrm{l})$ were dried in a glass scintillation vial under $\mathrm{N}_{2}$ gas and dissolved in $750 \mu \mathrm{l}$ 1:1 chloroform/methanol (both from Sigma-Aldrich). Then $250 \mu$ l boron trifluoride-methanol (Sigma-Aldrich) was added, and the vial was heated to $100^{\circ} \mathrm{C}$ for 5 minutes. After cooling, $1 \mathrm{ml}$ pentane was added and the samples were vortexed for 1 minute, then centrifuged at $2000 \mathrm{~g}$ for 5 minutes. The upper layer was transferred to a new glass vial using a Pasteur pipette and dried under $\mathrm{N}_{2}$ gas. The sample was then resuspended in $100 \mu$ hexane (Sigma-Aldrich), and enrichment was measured by GC/MS (CI mode, m/z $271[\mathrm{~m}+0]$ and $287[\mathrm{~m}+16]$ ).

For all metabolites, turnover was calculated using the following equation: turnover $=[$ (tracer enrichment/plasma enrichment $)-1]$ $\times$ infusion rate.

Hormones and substrates. Plasma glucose concentrations were measured enzymatically using the YSI Glucose Analyzer. Plasma insu- lin (Mercodia; 10-1250 [rat] and 10-1247 [mouse]), leptin (Abcam; ab100773 [rat] and ab100718 [mouse]), corticosterone (Abcam; ab108821), ACTH (MyBioSource; MBS2502683), epinephrine (Abnova; KA1877), norepinephrine (Abnova; KA1877), growth hormone (Millipore; EZRMGH-45K), IGF-1 (R\&D Systems; MG100), and glucagon (Sigma-Aldrich; RAB0202) concentrations were measured by ELISA. Plasma NEFA concentrations were measured spectrophotometrically using the Wako Diagnostics NEFA Kit (HR Series NEFA-HR; ref. 2). Plasma $\beta \mathrm{OHB}$, sodium, potassium, and chloride concentrations were measured by COBAS. The anion gap was measured using the following equation: anion gap $=\left[\mathrm{Na}^{+}\right]+\left[\mathrm{K}^{+}\right]-\left(\left[\mathrm{HCO}_{3}^{-}\right]+\left[\mathrm{Cl}^{-}\right]\right)$. Plasma acetate concentrations were measured by GC/MS: $25 \mu \mathrm{l}$ plasma was spiked with an equal volume of $\left[{ }^{2} \mathrm{H}\right]$ sodium acetate $(0.5 \mathrm{mM})$ and acidified with $50 \mu \mathrm{l} 1 \mathrm{M} \mathrm{HCl}$. After shaking for 20 minutes, samples were derivatized with $40 \mu \mathrm{mol}$ 1,3-dicyclohexylcarlodiimide and $40 \mu \mathrm{mol}$ 2,4-difluoroaniline (both from Sigma-Aldrich), shaken for 1 hour, and dried under $\mathrm{N}_{2}$ gas. Samples were then resuspended in ethyl acetate (Sigma-Aldrich), and peak areas $(\mathrm{m} / \mathrm{z} 171[\mathrm{~m}+0]$ and $174[\mathrm{~m}+4])$ were measured by GC/MS (EI mode).

Hepatic acetyl and malonyl CoA concentrations were measured by liquid chromatography-MS/MS (LC-MS/MS) as described (15). Liver and skeletal muscle triglyceride content were measured in diabetic mice enzymatically using the method of Bligh and Dyer (31), and ceramide concentrations were measured by LC-MS/MS as previously described (32).

Statistics. All statistical analysis was performed and figures were generated using GraphPad Prism. Comparisons of 2 groups were performed using the 2-tailed unpaired Student's $t$ test, and comparisons of 3 or 4 groups were performed using Bonferroni's multiple comparisons test.

Study approval. All animal experiments were approved by the Yale University Institutional Animal Care and Use Committee.

\section{Author contributions}

RJP and GIS designed the study. All authors performed experiments and analyzed data. The manuscript was written by RJP and GIS with input from all authors.

\section{Acknowledgments}

We thank Jianying Dong, Xian-Man Zhang, Mario Kahn, Irina Smolgovsky, John Stack, Gina Butrico, and Ali Nasiri for their expert technical assistance. This study was funded by the following grants from the US Public Health Service: R01 DK40936, U2C DK059635, P30 DK45735, T32 DK101019.

Address correspondence to: Gerald I. Shulman, PO Box 208020, 333 Cedar Street, New Haven, Connecticut, 06520-8020, USA. Phone: 203.785.5447; E-mail: gerald.shulman@yale.edu.
1. Yu X, Park BH, Wang MY, Wang ZV, Unger RH. Making insulin-deficient type 1 diabetic rodents thrive without insulin. Proc Natl Acad Sci U S A. 2008;105(37):14070-14075.

2. Fujikawa T, Chuang JC, Sakata I, Ramadori G, Coppari R. Leptin therapy improves insulindeficient type 1 diabetes by CNS-dependent mechanisms in mice. Proc Natl Acad Sci US A. 2010;107(40):17391-17396.

3. Hidaka S, et al. Chronic central leptin infusion restores hyperglycemia independent of food intake and insulin level in streptozotocin-induced diabetic rats. FASEB J. 2002;16(6):509-518.

4. Lin CY, Higginbotham DA, Judd RL, White BD. Central leptin increases insulin sensitivity in streptozotocin-induced diabetic rats. Am J Physiol Endocrinol Metab. 2002;282(5):E1084-E1091.

5. Fujikawa T, et al. Leptin engages a hypothalamic neurocircuitry to permit survival in the absence of insulin. Cell Metab. 2013;18(3):431-444.
6. Meek TH, et al. Leptin action in the ventromedial hypothalamic nucleus is sufficient, but not necessary, to normalize diabetic hyperglycemia Endocrinology. 2013;154(9):3067-3076.

7. Chinookoswong N, Wang JL, Shi ZQ. Leptin restores euglycemia and normalizes glucose turnover in insulin-deficient diabetes in the rat. Diabetes. 1999;48(7):1487-1492.

8. Wang MY, et al. Leptin therapy in insulin-deficient type I diabetes. Proc Natl Acad Sci US A. 
2010;107(11):4813-4819.

9. Park JY, et al. Type 1 diabetes associated with acquired generalized lipodystrophy and insulin resistance: the effect of long-term leptin therapy. JClin Endocrinol Metab. 2008;93(1):26-31.

10. Xu Y, Chang JT, Myers MG, Xu Y, Tong Q. Euglycemia restoration by central leptin in type 1 diabetes requires STAT3 signaling but not fast-acting neurotransmitter release. Diabetes. 2016;65(4):1040-1049.

11. Foster DW. Malonyl-CoA: the regulator of fatty acid synthesis and oxidation. JClin Invest. 2012;122(6):1958-1959.

12. Perry RJ, et al. Leptin reverses diabetes by suppression of the hypothalamic-pituitary-adrenal axis. Nat Med. 2014;20(7):759-763.

13. Morton GJ, Meek TH, Matsen ME, Schwartz MW. Evidence against hypothalamic-pituitary-adrenal axis suppression in the antidiabetic action of leptin. JClin Invest. 2015;125(12):4587-4591.

14. Mayer N, et al. Development of small-molecule inhibitors targeting adipose triglyceride lipase. Nat Chem Biol. 2013;9(12):785-787.

15. Perry RJ, et al. Hepatic acetyl CoA links adipose tissue inflammation to hepatic insulin resistance and type 2 diabetes. Cell. 2015;160(4):745-758.

16. McGarry JD, Mannaerts GP, Foster DW. A possible role for malonyl-CoA in the regulation of hepatic fatty acid oxidation and ketogenesis. JClin Invest. 1977;60(1):265-270.
17. Perry RJ, Lee S, Ma L, Zhang D, Schlessinger J, Shulman GI. FGF1 and FGF19 reverse diabetes by suppression of the hypothalamic-pituitaryadrenal axis. Nat Commun. 2015;6:6980.

18. Petersen KF, et al. Leptin reverses insulin resistance and hepatic steatosis in patients with severe lipodystrophy. J Clin Invest. 2002;109(10):1345-1350.

19. Shulman GI. Ectopic fat in insulin resistance, dyslipidemia, and cardiometabolic disease. $N$ Engl $J$ Med. 2014;371(12):1131-1141.

20. Samuel VT, Shulman GI. The pathogenesis of insulin resistance: integrating signaling pathways and substrate flux. JClin Invest. 2016;126(1):12-22.

21. Petersen MC, et al. Insulin receptor Thr1160 phosphorylation mediates lipid-induced hepatic insulin resistance. J Clin Invest. 2016;126(11):4361-4371.

22. Ruud J, Brüning JC. Metabolism: Light on leptin link to lipolysis. Nature. 2015;527(7576):43-44.

23. Cummings BP, et al. Subcutaneous administration of leptin normalizes fasting plasma glucose in obese type 2 diabetic UCD-T2DM rats. Proc Natl Acad Sci U S A. 2011;108(35):14670-14675.

24. Denroche HC, et al. Leptin therapy reverses hyperglycemia in mice with streptozotocininduced diabetes, independent of hepatic leptin signaling. Diabetes. 2011;60(5):1414-1423.

25. Kim GH, Szabo A, King EM, Ayala J, Ayala JE, Altarejos JY. Leptin recruits Creb-regulated transcriptional coactivator 1 to improve hyperglycemia in insulin-deficient diabetes. Mol Metab. 2015;4(3):227-236.

26. Rossetti L, Shulman GI, Zawalich W, DeFronzo RA. Effect of chronic hyperglycemia on in vivo insulin secretion in partially pancreatectomized rats. JClin Invest. 1987;80(4):1037-1044.

27. Rossetti L, Smith D, Shulman GI, Papachristou D, DeFronzo RA. Correction of hyperglycemia with phlorizin normalizes tissue sensitivity to insulin in diabetic rats. J Clin Invest. 1987;79(5):1510-1515.

28. Layman DK, Wolfe RR. Sample site selection for tracer studies applying a unidirectional circulatory approach. Am JPhysiol. 1987;253(2 Pt 1):E173-E178.

29. Katz J. On the determination of turnover in vivo with tracers. Am J Physiol. 1992; 263(3 Pt 1):E417-E424.

30. Katz J, Okajima F, Chenoweth M, Dunn A. The determination of lactate turnover in vivo with $3 \mathrm{H}$ - and 14C-labelled lactate. The significance of sites of tracer administration and sampling. Biochem J. 1981;194(2):513-524.

31. Bligh EG, Dyer WJ. A rapid method of total lipid extraction and purification. Can J Biochem Physiol. 1959;37(8):911-917.

32. Yu C, et al. Mechanism by which fatty acids inhibit insulin activation of insulin receptor substrate-1 (IRS-1)-associated phosphatidylinositol 3-kinase activity in muscle. J Biol Chem. 2002;277(52):50230-50236. 